\title{
Recursive methods for a multi-dimensional risk process with common shocks
}

\author{
Lan Gong*, Andrei L. Badescu* and Eric C.K. Cheung ${ }^{\dagger}$
}

January 12, 2012

\begin{abstract}
In this paper, a multi-dimensional risk model with common shocks is studied. Using a simple probabilistic approach via observing the risk processes at claim instants, recursive integral formulas are developed for the survival probabilities as well as for a class of GerberShiu expected discounted penalty functions that include the surplus levels at ruin. Under the assumption of exponential or mixed Erlang claims, the recursive integrals can be simplified to give recursive sums which are computationally more tractable. Numerical examples including an optimal capital allocation problem are also given towards the end.
\end{abstract}

Keywords: Common shock, Deficit at ruin; Gerber-Shiu expected discounted penalty function; Recursive methods; Survival probability; Multi-dimensional risk process; Optimal capital allocation.

\section{Introduction}

Multi-dimensional risk theory gained a lot of attention in the past few years mainly due to the complexity of the problems and the lack of closed-form results even under very basic model assumptions. This paper continues the search for new results for a class of multi-dimensional risk processes where the claim arrivals in $M$ classes of business are assumed to be dependent Poisson processes with common shocks. In this paper, the surplus process $\left\{U_{i}(t)\right\}_{t \geq 0}$ of the $i$-th class of business is described by, for $i=1,2, \ldots, M$,

$$
U_{i}(t)=u_{i}+c_{i} t-\sum_{k=1}^{N_{i i}(t)} Y_{i, k}-\sum_{k=1}^{N_{c}(t)} Z_{i, k}, \quad t \geq 0,
$$

* Department of Statistics, University of Toronto, 100 St. George St., Toronto, Ontario, Canada

${ }^{\dagger}$ Department of Statistics and Actuarial Science, University of Hong Kong, Pokfulam, Hong Kong 
with the associated initial capital and premium rate denoted by $u_{i}=U_{i}(0) \geq 0$ and $c_{i}>0$ respectively. We adopt the usual convention that $\sum_{i=j}^{k}=0$ for any $j>k$, and since we are interested in multivariate process, it is assumed that $m>1$. The counting processes $\left\{N_{11}(t)\right\}_{t \geq 0},\left\{N_{22}(t)\right\}_{t \geq 0}, \ldots,\left\{N_{M M}(t)\right\}_{t \geq 0}$ and $\left\{N_{c}(t)\right\}_{t \geq 0}$ are Poisson processes with rates $\lambda_{11}$, $\lambda_{22}, \ldots, \lambda_{M M}$ and $\lambda_{c}$ respectively. For a given $i=1,2, \ldots, M,\left\{Y_{i, k}\right\}_{k=1}^{\infty}$ forms an independent and identically distributed (i.i.d.) sequence of positive random variables with common density function $f_{i i}\left(x_{i}\right)$. Furthermore, $\left\{\left(Z_{1, k}, Z_{2, k}, \ldots, Z_{M, k}\right)\right\}_{k=1}^{\infty}$ is a sequence of i.i.d. $M$ dimensional positive random vectors with common joint density function $f_{c}\left(x_{1}, x_{2}, \ldots, x_{M}\right)$. It is further assumed that $\left\{N_{i i}(t)\right\}_{t \geq 0}(i=1,2, \ldots, M),\left\{N_{c}(t)\right\}_{t \geq 0},\left\{Y_{i, k}\right\}_{k=1}^{\infty}(i=1,2, \ldots, M)$ and $\left\{\left(Z_{1, k}, Z_{2, k}, \ldots, Z_{M, k}\right)\right\}_{k=1}^{\infty}$ are all mutually independent. The time of ruin for the $i$-th risk process is defined by $\tau_{i}=\inf \left\{t \geq 0 \mid U_{i}(t)<0\right\}$ for $i=1,2, \ldots, M$. Moreover, the positive security loading condition which makes sure ruin is not a certain event in the $i$-th process is given by $\theta_{i}>0$, where $c_{i}=\left(1+\theta_{i}\right)\left(\lambda_{i i} E\left[Y_{i, 1}\right]+\lambda_{c} E\left[Z_{i, 1}\right]\right)$.

The model with the dynamics described in (1) has the following interpretation:

1. For each fixed $i=1,2, \ldots, M$, the process $\left\{N_{i i}(t)\right\}_{t \geq 0}$ counts the number of claims faced by the $i$-th business line up to time $t$, arising from the 'usual' claim occurrences which only induce claims in one line but not the others. Under such 'usual' claim occurrences, the resulting claim sizes are given by the sequence $\left\{Y_{i, k}\right\}_{k=1}^{\infty}$.

2. The process $\left\{N_{c}(t)\right\}_{t \geq 0}$ counts the number of 'common shocks' faced by the $M$ lines. The common shock component can be interpreted as a natural disaster that causes different kinds of claims, or as a claim that affects all $M$ lines of business at the same time. The $k$-th common shock results in a claim of size $Z_{i, k}$ in the $i$-th business line, and the claim sizes $Z_{1, k}, Z_{2, k}, \ldots$, and $Z_{M, k}$ are possibly dependent variables.

In the case where $f_{c}\left(x_{1}, x_{2}, \ldots, x_{M}\right)=\prod_{i=1}^{M} f_{i i}\left(x_{i}\right)$, for each fixed $k=1,2, \ldots$ the variables $Z_{1, k}, Z_{2, k}, \ldots$, and $Z_{M, k}$ are independent with the same marginal distributions as $Y_{1, k}, Y_{2, k}, \ldots$, and $Y_{M, k}$ respectively. Then the process (1) can be equivalently represented as, for $i=1,2, \ldots, M$,

$$
U_{i}(t)=u_{i}+c_{i} t-\sum_{k=1}^{N_{i}(t)} X_{i, k}, \quad t \geq 0,
$$

where $\left\{N_{1}(t)\right\}_{t \geq 0},\left\{N_{2}(t)\right\}_{t \geq 0}, \ldots$, and $\left\{N_{M}(t)\right\}_{t \geq 0}$ are (common shock) correlated Poisson processes at rates $\lambda_{1}, \lambda_{2}, \ldots$, and $\lambda_{M}$ respectively. More specifically, for $i=1,2, \ldots, M$, one has that $N_{i}(t)=N_{i i}(t)+N_{c}(t)$ where $\lambda_{i}=\lambda_{i i}+\lambda_{c}$, and $\left\{N_{i}(t)\right\}_{t \geq 0}$ is independent of the i.i.d. sequence $\left\{X_{i, k}\right\}_{k=1}^{\infty}$ with common density $f_{i}\left(x_{i}\right) \equiv f_{i i}\left(x_{i}\right)$. The positive security loading condition reduces to $c_{i}>\lambda_{i} E\left[X_{i, 1}\right]$. The model (2) will also be discussed in this paper.

As pointed out in Chan et al. (2003), the concept of 'ruin' in the multi-dimensional setting has different meanings and interpretations when compared to the standard univariate risk case. For instance, $T_{\text {or }}=\inf \left\{t \geq 0 \mid \min \left\{U_{1}(t), U_{2}(t), \ldots, U_{M}(t)\right\}<0\right\}=\min \left(\tau_{1}, \tau_{2}, \ldots, \tau_{M}\right)$ denotes the first time when (at least) one of $\left\{U_{1}(t)\right\}_{t \geq 0},\left\{U_{2}(t)\right\}_{t \geq 0}, \ldots$, or $\left\{U_{M}(t)\right\}_{t \geq 0}$ is below zero; whereas $T_{\text {sim }}=\inf \left\{t \geq 0 \mid \max \left\{U_{1}(t), U_{2}(t), \ldots, U_{M}(t)\right\}<0\right\}$ denotes the first time that all $\left\{U_{1}(t)\right\}_{t \geq 0},\left\{U_{2}(t)\right\}_{t \geq 0}, \ldots$, and $\left\{U_{M}(t)\right\}_{t \geq 0}$ are below zero simultaneously. In the bivariate 
case (i.e. $M=2$ ), some authors (e.g. Avram et al. (2008a,b) and Rabehasaina (2009)) refer to $T_{\text {or }}$ and $T_{\text {sim }}$ as the first exit from the positive quadrant and the first entrance into the negative quadrant respectively. Other ruin concepts include $T_{\text {and }}=\max \left(\tau_{1}, \tau_{2}, \ldots, \tau_{M}\right)$ which denotes the first time when ruin has occurred in all $\left\{U_{1}(t)\right\}_{t \geq 0},\left\{U_{2}(t)\right\}_{t \geq 0}, \ldots$, and $\left\{U_{M}(t)\right\}_{t \geq 0}$, but not necessarily simultaneously; and $T_{\text {sum }}=\inf \left\{t \geq 0 \mid \sum_{i=1}^{M} U_{i}(t)<0\right\}$ which is the first time that the sum of $\left\{U_{1}(t)\right\}_{t \geq 0},\left\{U_{2}(t)\right\}_{t \geq 0}, \ldots$, and $\left\{U_{M}(t)\right\}_{t \geq 0}$ becomes negative. Historically, it is known that $T_{\text {sum }}$ is relatively easy to be analyzed, as the problem can be reduced to a onedimensional ruin problem. Chan et al. (2003) derived an explicit formula for the infinite-time ruin probability for $T_{\text {sum }}$ when $M=2$, in a model with common shocks only (i.e. $\lambda_{11}=\lambda_{22}=0$ ), assuming that the claims in the two lines are independent phase-type random variables. Later on, Cai and Li (2005) generalized their result to a multi-dimensional setting in which claims are introduced through a multivariate phase-type structure. In contrast, when one refers to $T_{\text {or }}, T_{\text {sim }}$ or $T_{\text {and }}$, things change drastically, and the existing results consist mainly of bounds, approximations and asymptotics. Various asymptotics and large deviation results were derived by Collamore $(1996,1998)$ in a multi-dimensional risk model as an extension of the Cramér's asymptotic ruin formula. Simple lower and upper bounds for the ruin probabilities in the cases of $T_{\text {or }}$ and $T_{\text {sim }}$ were obtained by Chan et al. (2003) when $M=2$, complemented by various (stochastic) bounds in Cai and $\operatorname{Li}(2005,2007)$ for all three definitions of ruin in the multidimensional case. For $T_{\text {or }}$, Yuen et al. (2006) obtained an approximation to the finite-time survival probability in the continuous bivariate compound Poisson model (2) via the introduction of a discrete bivariate compound binomial equivalent, and derived an upper bound for the ruin probability. Using martingale techniques, Li et al. (2007) also derived upper bounds for the infinite-time ruin probability, as well as asymptotic results for the finite-time ruin probability associated to $T_{\text {sim }}$ in a two-dimensional perturbed risk model.

In addition to the above papers, a few exact results are also available in the literature of bivariate risk processes. In a model with common shocks only, Chan et al. (2003) obtained a partial integro-differential equation (PIDE) for the survival probability $\Phi\left(u_{1}, u_{2}\right)=\operatorname{Pr}\left(T_{\text {or }}=\right.$ $\left.\infty \mid\left(U_{1}(0), U_{2}(0)\right)=\left(u_{1}, u_{2}\right)\right)$ using the usual renewal approach. Then, they derived an expression for the double Laplace transform with respect to $\left(u_{1}, u_{2}\right)$, but did not invert it. Assuming exponentially distributed claims in both lines of business, the afore-mentioned PIDE was solved by Dang et al. (2009), who showed that the unique solution can be calculated as $\Phi\left(u_{1}, u_{2}\right)=\lim _{n \rightarrow \infty} \Phi_{n}\left(u_{1}, u_{2}\right)$, where $\Phi_{n}\left(u_{1}, u_{2}\right)$ is expressed in terms of $\Phi_{n-1}(\cdot, \cdot)$ as recursive integrals (see Remark 1). This is also complemented by some recursive integral formulas in Rabehasaina (2009, Section 5) using fluid flow techniques and the idea of embedding it in a multi-dimensional model under a Markovian environment and possibly interest force. In the very specific model (1) in which $\lambda_{11}=\lambda_{22}=0$ and $(1-a) Z_{1, k}=a Z_{2, k}$ for $k=1,2, \ldots$ where $a \in(0,1)$, Avram et al. (2008a,b) derived the ruin probabilities for $T_{\text {or }}, T_{\text {sim }}$ and $T_{\text {and }}$. Their method is based on the reduction of the bivariate problem to various univariate problems which is possible due to proportionality of claims. Note that such a model has the practical interpretation that the two lines of business are an insurer and a reinsurer who are engaged in a proportional reinsurance contract. Some of their results are further extended by Badescu et al. (2011) who obtained the Laplace transform of $T_{\text {or }}$ by allowing for $\lambda_{11}>0$. Interested readers are also referred to Collamore (2002) for important sampling techniques for multivariate ruin problems as well as Czarna and Palmowski (2011) for dividend problems in a bivariate risk model with proportional reinsurance. See also Asmussen and Albrecher (2010, Chapter XIII.9) for an overview of multi-dimensional risk processes and additional references. 
In this paper, we mainly focus on the study of ruin-related quantities defined for the ruin time $T_{\text {or }}$. The goal of this paper is two-fold. First, we present general recursive integral formulas in the general $M$-dimensional risk process (1) via the idea of observing the process at claim instants, for both survival probability and a novel quantity known as the Gerber-Shiu function (Gerber and Shiu (1998)) for ruin at the $n$-th claim instant. These will be the contents of Sections 2 and 3 respectively. Second, motivated by the difficulty and instability to obtain numerical results from the direct application of recursive integral formulas, we show that in certain cases the recursive integrals can be simplified to give recursive sums (e.g. Stanford and Stroiński (1994) and Stanford et al. (2000)) which are computationally more tractable. To this end, in Section 4 we derive examples for the survival probability and the expected discounted deficit at ruin under the assumptions of both exponential and mixed Erlang claims in the bivariate case. Section 5 gives an account for the survival probability in the $T_{\text {and }}$ case in relation to $T_{\text {or }}$. Section 6 is concerned with numerical illustrations of the results obtained in Sections 4 and 5. In particular, a capital allocation problem in which the two lines of business split among them a fixed amount of the total initial surplus in order to minimize the ruin probability for $T_{\text {or }}$ and $T_{\text {and }}$ is considered (see also Remark 2). Section 7 ends the paper by some concluding remarks.

\section{A recursive approach to survival probability}

In this section, we are interested in the survival probability for $T_{\text {or }}$ pertaining to the $M$-dimensional risk process (1) under the general framework, which is formally defined as

$\Phi\left(u_{1}, u_{2}, \ldots, u_{M}\right)=\operatorname{Pr}{ }_{\left(u_{1}, u_{2}, \ldots, u_{M}\right)}\left(T_{\text {or }}=\infty\right)=\operatorname{Pr}{ }_{\left(u_{1}, u_{2}, \ldots, u_{M}\right)}\left(U_{i}(t) \geq 0\right.$ for all $\left.t \geq 0 ; i=1,2, \ldots, M\right)$,

where $\operatorname{Pr}{ }_{\left(u_{1}, u_{2}, \ldots, u_{M}\right)}$ denotes the probability law given the initial conditions $\left(U_{1}(0), U_{2}(0), \ldots, U_{M}(0)\right)=$ $\left(u_{1}, u_{2}, \ldots, u_{M}\right)$. Then $\Psi\left(u_{1}, u_{2}, \ldots, u_{M}\right)=1-\Phi\left(u_{1}, u_{2}, \ldots, u_{M}\right)$ represents the associated joint ruin probability.

Since our idea is to look recursively at the survival probabilities at each claim instant, for $n=0,1, \ldots$, we introduce the $n$-th claim event arrival time $S_{n}=\sum_{k=1}^{n} V_{k}$. Here $\left\{V_{k}\right\}_{k=1}^{\infty}$ are the inter-arrival times corresponding to the Poisson process $\left\{\sum_{i=1}^{M} N_{i i}(t)+N_{c}(t)\right\}_{t \geq 0}$ and hence i.i.d. exponential random variables each with mean $1 / \lambda_{s}$, where $\lambda_{s}=\sum_{i=1}^{M} \lambda_{i i}+\lambda_{c}$. Then for $n=0,1, \ldots$, we define $\Phi_{n}\left(u_{1}, u_{2}, \ldots, u_{M}\right)$ to be the probability that all $\left\{U_{1}(t)\right\}_{t \geq 0},\left\{U_{2}(t)\right\}_{t \geq 0}, \ldots$, and $\left\{U_{M}(t)\right\}_{t \geq 0}$ survive up to and including the $n$-th claim, namely

$$
\begin{aligned}
\Phi_{n}\left(u_{1}, u_{2}, \ldots, u_{M}\right) & =\operatorname{Pr}{ }_{\left(u_{1}, u_{2}, \ldots, u_{M}\right)}\left(T_{\text {or }}>S_{n}\right) \\
& =\operatorname{Pr}{ }_{\left(u_{1}, u_{2}, \ldots, u_{M}\right)}\left(U_{i}(t) \geq 0 \text { for all } 0 \leq t \leq S_{n} ; i=1,2, \ldots, M\right) \\
& =\operatorname{Pr}{ }_{\left(u_{1}, u_{2}, \ldots, u_{M}\right)}\left(U_{i}\left(S_{k}\right) \geq 0 \text { for all } k=0,1, \ldots, n ; i=1,2, \ldots, M\right),
\end{aligned}
$$

with $\Psi_{n}\left(u_{1}, u_{2}, \ldots, u_{M}\right)=1-\Phi_{n}\left(u_{1}, u_{2}, \ldots, u_{M}\right)$ being the associated joint ruin probability.

Conditioning on the time of the first claim event and the resulting claim sizes in the $M$ lines 
(if any), one can recursively write, for $n=0,1, \ldots$,

$$
\begin{aligned}
& \Phi_{n+1}\left(u_{1}, \ldots, u_{M}\right) \\
= & \sum_{i=1}^{M} \int_{0}^{\infty} \int_{0}^{u_{i}+c_{i} t} \Phi_{n}\left(u_{1}+c_{1} t, \ldots, u_{i-1}+c_{i-1} t, u_{i}+c_{i} t-x_{i}, u_{i+1}+c_{i+1} t, \ldots, u_{M}+c_{M} t\right) f_{i i}\left(x_{i}\right) \lambda_{i i} e^{-\lambda_{s} t} d x_{i} d t \\
& +\int_{0}^{\infty} \int_{0}^{u_{M}+c_{M} t} \cdots \int_{0}^{u_{1}+c_{1} t} \Phi_{n}\left(u_{1}+c_{1} t-x_{1}, \ldots, u_{M}+c_{M} t-x_{M}\right) f_{c}\left(x_{1}, \ldots, x_{M}\right) \lambda_{c} e^{-\lambda_{s} t} d x_{1} \cdots d x_{M} d t .
\end{aligned}
$$

The starting point of the above recursive scheme is

$$
\Phi_{0}\left(u_{1}, u_{2}, \ldots, u_{M}\right)=1
$$

since ruin is impossible without any claims. The ultimate survival probability for $T_{\text {or }}$ is then given by the limit

$$
\Phi\left(u_{1}, u_{2}, \ldots, u_{M}\right)=\lim _{n \rightarrow \infty} \Phi_{n}\left(u_{1}, u_{2}, \ldots, u_{M}\right) .
$$

The next example provides an illustration of the simplifications that occur in the simplest bivariate case where claims are exponentially distributed.

Example 1 We consider the bivariate version of model (2) under exponential claims with densities $f_{i}\left(x_{i}\right)=\mu_{i} e^{-\mu_{i} x_{i}}$ for $i=1,2$. We shall use the convention $\lambda_{c}=\lambda_{12}$ for bivariate risk process. By plugging $n=0$ into (3) with the use of the starting value $\Phi_{0}\left(u_{1}, u_{2}\right)=1$, we obtain

$$
\Phi_{1}\left(u_{1}, u_{2}\right)=1-\frac{\lambda_{s}-\lambda_{22}}{\lambda_{s}+c_{1} \mu_{1}} e^{-\mu_{1} u_{1}}-\frac{\lambda_{s}-\lambda_{11}}{\lambda_{s}+c_{2} \mu_{2}} e^{-\mu_{2} u_{2}}+\xi e^{-\left(\mu_{1} u_{1}+\mu_{2} u_{2}\right)},
$$

where $\xi=\lambda_{12} /\left(\lambda_{s}+c_{1} \mu_{1}+c_{2} \mu_{2}\right)$. In general, for $n=1,2, \ldots$, (3) reduces to

$$
\begin{aligned}
& \Phi_{n+1}\left(u_{1}, u_{2}\right) \\
= & \int_{0}^{\infty} \int_{0}^{u_{1}+c_{1} t} \Phi_{n}\left(u_{1}+c_{1} t-x_{1}, u_{2}+c_{2} t\right) \mu_{1} e^{-\mu_{1} x_{1}} \lambda_{11} e^{-\lambda_{s} t} d x_{1} d t \\
& +\int_{0}^{\infty} \int_{0}^{u_{2}+c_{2} t} \Phi_{n}\left(u_{1}+c_{1} t, u_{2}+c_{2} t-x_{2}\right) \mu_{2} e^{-\mu_{2} x_{2}} \lambda_{22} e^{-\lambda_{s} t} d x_{2} d t \\
& +\int_{0}^{\infty} \int_{0}^{u_{2}+c_{2} t} \int_{0}^{u_{1}+c_{1} t} \Phi_{n}\left(u_{1}+c_{1} t-x_{1}, u_{2}+c_{2} t-x_{2}\right) \mu_{1} e^{-\mu_{1} x_{1}} \mu_{2} e^{-\mu_{2} x_{2}} \lambda_{12} e^{-\lambda_{s} t} d x_{1} d x_{2} d t \\
= & \xi \mu_{1} \mu_{2} \int_{0}^{u_{1}} \int_{0}^{u_{2}} \Phi_{n}\left(a_{1}, a_{2}\right) e^{\mu_{1}\left(a_{1}-u_{1}\right)} e^{\mu_{2}\left(a_{2}-u_{2}\right)} d a_{2} d a_{1} \\
& +\left(\xi \mu_{1} \mu_{2}+\frac{\lambda_{22} \mu_{2}}{c_{1}}\right) \int_{u_{1}}^{\infty} \int_{0}^{\frac{c_{2}}{c_{1}}\left(a_{1}-u_{1}\right)+u_{2}} \Phi_{n}\left(a_{2}\right) e^{\lambda_{s}\left(\frac{u_{1}-a_{1}}{c_{1}}\right)} e^{\left.\left.-\mu_{2}\right) e^{\lambda_{s}\left(\frac{u_{2}-a_{2}}{c_{2}}\right)} e^{-\mu_{1}\left[u_{1}+\frac{c_{2}}{c_{1}}\left(a_{1}-u_{1}\right)-a_{2}\right]} d a_{2} d a_{1}\left(a_{2}-u_{2}\right)-a_{1}\right]} d a_{1} d a_{2}, \\
& +\left(\xi \mu_{1} \mu_{2}+\frac{\lambda_{11} \mu_{1}}{c_{2}}\right) \int_{u_{2}}^{\infty} \int_{0}^{\frac{c_{1}}{c_{2}}\left(a_{2}-u_{2}\right)+u_{1}}
\end{aligned}
$$

where the last line follows from various changes of variables and changes of order of integrations (the calculations are tedious but straightforward, the details being omitted here). Finally, the survival probability $\Phi\left(u_{1}, u_{2}\right)$ can be recursively calculated as $\Phi\left(u_{1}, u_{2}\right)=\lim _{n \rightarrow \infty} \Phi_{n}\left(u_{1}, u_{2}\right)$. 
Remark 1 If we let $\lambda_{11}=\lambda_{22}=0$ and $\lambda_{12}=\lambda$, then Example 1 reduces to Theorem 3.1 of Dang et al. (2009) who used a tedious proof based on the theory of PIDEs.

Example 2 In this example we consider a bivariate risk model (2) with common shocks only (i.e. $\lambda_{11}=\lambda_{22}=0$ and $\lambda_{c}=\lambda_{12}>0$ ) under phase-type (PH) distributed claim amounts. More precisely, we let $\left\{X_{1, k}\right\}_{k=1}^{\infty}$ and $\left\{X_{2, k}\right\}_{k=1}^{\infty}$ follow independent $P H$ distributions with parameters $(\boldsymbol{\alpha}, \boldsymbol{T})$ and $(\boldsymbol{\beta}, \boldsymbol{Q})$, so that the corresponding density functions are given by $f_{1}\left(x_{1}\right)=\boldsymbol{\alpha} e^{\boldsymbol{T} x_{1}} \boldsymbol{t}$ and $f_{2}\left(x_{2}\right)=\boldsymbol{\beta} e^{Q x_{2}} \boldsymbol{q}$ respectively. Using the notions of Kronecker product and Kronecker sum (see Asmussen and Albrecher (2010, Appendix A4)), one can perform integration on (3) which now involves matrix exponentials. Omitting the details, we obtain for $n=0,1, \ldots$,

$$
\begin{aligned}
& \Phi_{n+1}\left(u_{1}, u_{2}\right) \\
= & \int_{0}^{\infty} \int_{0}^{u_{1}+c_{1} t} \int_{0}^{u_{2}+c_{2} t} \Phi_{n}\left(u_{1}+c_{1} t-x_{1}, u_{2}+c_{2} t-x_{2}\right) \lambda_{12} e^{-\lambda_{12} t}\left(\boldsymbol{\alpha} e^{\boldsymbol{T} x_{1}} \boldsymbol{t}\right)\left(\boldsymbol{\beta} e^{\boldsymbol{Q} x_{2}} \boldsymbol{q}\right) d x_{2} d x_{1} d t \\
= & \int_{0}^{\infty} \int_{0}^{u_{1}+c_{1} t} \int_{0}^{u_{2}+c_{2} t} \Phi_{n}\left(a_{1}, a_{2}\right) \lambda_{12} e^{-\lambda_{12} t}\left(\boldsymbol{\alpha} e^{\boldsymbol{T}\left(u_{1}+c_{1} t-a_{1}\right)} \boldsymbol{t}\right)\left(\boldsymbol{\beta} e^{\boldsymbol{Q}\left(u_{2}+c_{2} t-a_{2}\right)} \boldsymbol{q}\right) d a_{2} d a_{1} d t \\
= & \int_{0}^{u_{1}} \int_{0}^{u_{2}} \Phi_{n}\left(a_{1}, a_{2}\right) \lambda_{12}(\boldsymbol{\alpha} \otimes \boldsymbol{\beta})\left[\lambda_{12} \oplus\left(-c_{1} \boldsymbol{T}\right) \oplus\left(-c_{2} \boldsymbol{Q}\right)\right]^{-1} e^{\left[\boldsymbol{T}\left(u_{1}-a_{1}\right)\right] \oplus\left[\boldsymbol{Q}\left(u_{2}-a_{2}\right)\right]}(\boldsymbol{t} \otimes \boldsymbol{q}) d a_{2} d a_{1} \\
& +\int_{u_{1}}^{\infty} \int_{0}^{\frac{c_{2}}{c_{1}\left(a_{1}-u_{1}\right)+u_{2}}} \Phi_{n}\left(a_{1}, a_{2}\right) \lambda_{12}(\boldsymbol{\alpha} \otimes \boldsymbol{\beta})\left[\lambda_{12} \oplus\left(-c_{1} \boldsymbol{T}\right) \oplus\left(-c_{2} \boldsymbol{Q}\right)\right]^{-1} e^{-\left[\lambda_{12} \oplus\left(-c_{1} \boldsymbol{T}\right) \oplus\left(-c_{2} \boldsymbol{Q}\right)\right]\left(\frac{a_{1}-u_{1}}{c_{1}}\right)} \\
& \times e^{\left[\boldsymbol{T}\left(u_{1}-a_{1}\right)\right] \oplus\left[\boldsymbol{Q}\left(u_{2}-a_{2}\right)\right]}(\boldsymbol{t} \otimes \boldsymbol{q}) d a_{2} d a_{1} \\
& +\int_{u_{2}}^{\infty} \int_{0}^{\frac{c_{1}}{c_{2}}\left(a_{2}-u_{2}\right)+u_{1}} \Phi_{n}\left(a_{1}, a_{2}\right) \lambda_{12}(\boldsymbol{\alpha} \otimes \boldsymbol{\beta})\left[\lambda_{12} \oplus\left(-c_{1} \boldsymbol{T}\right) \oplus\left(-c_{2} \boldsymbol{Q}\right)\right]^{-1} e^{-\left[\lambda_{12} \oplus\left(-c_{1} \boldsymbol{T}\right) \oplus\left(-c_{2} \boldsymbol{Q}\right)\right]\left(\frac{a_{2}-u_{2}}{c_{2}}\right)} \\
& \times e^{\left[\boldsymbol{T}\left(u_{1}-a_{1}\right)\right] \oplus\left[\boldsymbol{Q}\left(u_{2}-a_{2}\right)\right]}(\boldsymbol{t} \otimes \boldsymbol{q}) d a_{1} d a_{2},
\end{aligned}
$$

which can also be regarded as a generalization of Dang et al. (2009, Theorem 3.1) for phase-type distributed claim sizes without relying on the theory of PIDEs.

A natural question that arises from the recursive approach in this section is: can equations in the form of (3) be used to obtain numerical values for survival probabilities? To answer this, we ran several numerical examples in Mathematica and the computational time increases exponentially as $n$ increases. Using a MacBook Pro $2.13 \mathrm{GHz}$ Intel Core 2 Duo processor, it took us more than 48 hours to obtain a numerical value for $n=10$ in the simplest bivariate Example 1. Note also that source of potential numerical instability comes from the numerical integration that is used when evaluating (4). Such numerical problems arising from direct application of (3) will be addressed in Section 4, and in particular, Proposition 1 gives a computationally tractable expression for equation (3) in the case of mixed Erlang claims.

\section{Gerber-Shiu function for ruin at $n$-th claim instant}

This section aims at extending the results derived in the previous section to a subclass of the Gerber-Shiu expected discounted penalty function for ruin at the $n$-th claim instant (in the sense 
of $\left.T_{\text {or }}\right)$. For the $M$-dimensional risk process $\left\{\left(U_{1}(t), U_{2}(t), \ldots, U_{M}(t)\right)\right\}_{t \geq 0}$ under the general framework (1), if we are interested in the surplus levels at the ruin time $T_{\text {or }}$, a natural candidate of the Gerber-Shiu function (Gerber and Shiu (1998)) is given by

$$
\phi\left(u_{1}, u_{2}, \ldots, u_{M}\right)=E_{\left(u_{1}, u_{2}, \ldots, u_{M}\right)}\left[e^{-\delta T_{\text {or }}} w\left(U_{1}\left(T_{\text {or }}\right), U_{2}\left(T_{\text {or }}\right), \ldots, U_{M}\left(T_{\text {or }}\right)\right) I\left(T_{\text {or }}<\infty\right)\right],
$$

where $w\left(y_{1}, y_{2}, \ldots, y_{M}\right)$ is a penalty function that depends on the surplus levels at time $T_{\text {or }}$ in all the processes $\left\{U_{1}(t)\right\}_{t \geq 0},\left\{U_{2}(t)\right\}_{t \geq 0}, \ldots$, and $\left\{U_{M}(t)\right\}_{t \geq 0}, I(A)$ is the indicator function of the event $A$, and $E_{\left(u_{1}, u_{2}, \ldots, u_{M}\right)}$ represents the conditional expectation given the initial surplus levels $\left(U_{1}(0), U_{2}(0), \ldots, U_{M}(0)\right)=\left(u_{1}, u_{2}, \ldots, u_{M}\right)$. It is instructive to note that at time $T_{\text {or }}$ some lines of business may still possess non-negative surplus levels. More specifically, if ruin is caused by the occurrence of claims in one line only, say line $i$ (due to the Poisson process $\left\{N_{i i}(t)\right\}_{t \geq 0}$ ), then at time $T_{\text {or }}$ the surplus levels of all companies other than line $i$ are indeed non-negative. In contrast, if ruin is caused by the common shock component $\left\{N_{c}(t)\right\}_{t \geq 0}$, then it is possible that more than one of the $M$ lines are actually ruined together at time $T_{\text {or }}$. Therefore it makes sense to distinguish between cases where different subsets of the $M$ lines are ruined at time $T_{\text {or }}$. In what follows we consider a penalty function in the form of

$$
w\left(y_{1}, y_{2}, \ldots, y_{M}\right)=\sum_{A \in \Gamma} w^{A}\left(y_{1}, y_{2}, \ldots, y_{M}\right) I\left(y_{i}<0 \text { for } i \in A ; y_{j} \geq 0 \text { for } j \notin A\right),
$$

where $\Gamma$ contains all subsets of $\{1,2, \ldots, M\}$ excluding the empty set, and $w^{A}\left(y_{1}, y_{2}, \ldots, y_{M}\right)$ is a penalty function which possibly depends on the index set $A$. Hence, on the event set $\left\{T_{\text {or }}<\infty\right\}$,

$$
\begin{aligned}
& w\left(U_{1}\left(T_{\text {or }}\right), U_{2}\left(T_{\text {or }}\right), \ldots, U_{M}\left(T_{\text {or }}\right)\right) \\
= & \sum_{i=1}^{M} w^{\{i\}}\left(U_{1}\left(T_{\text {or }}\right), U_{2}\left(T_{\text {or }}\right), \ldots, U_{M}\left(T_{\text {or }}\right)\right) I\left(U_{i}\left(T_{\text {or }}\right)<0 ; U_{j}\left(T_{\text {or }}\right) \geq 0 \text { for } j \neq i\right) \\
& +\sum_{A \in \Gamma \backslash \cup_{k=1}^{M}\{k\}} w^{A}\left(U_{1}\left(T_{\text {or }}\right), U_{2}\left(T_{\text {or }}\right), \ldots, U_{M}\left(T_{\text {or }}\right)\right) I\left(U_{i}\left(T_{\text {or }}\right)<0 \text { for } i \in A ; U_{j}\left(T_{\text {or }}\right) \geq 0 \text { for } j \notin A\right) \\
= & \sum_{i=1}^{M} w^{\{i\}}\left(U_{1}\left(T_{\text {or }}\right), U_{2}\left(T_{\text {or }}\right), \ldots, U_{M}\left(T_{\text {or }}\right)\right) I\left(\tau_{i}=T_{\text {or }} ; \tau_{j}>T_{\text {or }} \text { for } j \neq i\right) \\
& +\sum_{A \in \Gamma \backslash \cup_{k=1}^{M}\{k\}} w^{A}\left(U_{1}\left(T_{\text {or }}\right), U_{2}\left(T_{\text {or }}\right), \ldots, U_{M}\left(T_{\text {or }}\right)\right) I\left(\tau_{i}=T_{\text {or }} \text { for } i \in A ; \tau_{j}>T_{\text {or }} \text { for } j \notin A\right) .
\end{aligned}
$$

With the penalty function given by (6) or (7), various information can be extracted from the Gerber-Shiu function (5) by appropriate choice of the functions $w^{A}\left(y_{1}, y_{2}, \ldots, y_{M}\right)$ for $A \in \Gamma$, as illustrated in the following example in the bivariate case.

Example 3 In the bivariate case $M=2$, the penalty function (7) reduces to

$$
\begin{aligned}
& w\left(U_{1}\left(T_{\text {or }}\right), U_{2}\left(T_{\text {or }}\right)\right) \\
= & w^{\{1\}}\left(U_{1}\left(T_{\text {or }}\right), U_{2}\left(T_{\text {or }}\right)\right) I\left(\tau_{1}<\tau_{2}\right)+w^{\{2\}}\left(U_{1}\left(T_{\text {or }}\right), U_{2}\left(T_{\text {or }}\right)\right) I\left(\tau_{2}<\tau_{1}\right)+w^{\{1,2\}}\left(U_{1}\left(T_{\text {or }}\right), U_{2}\left(T_{\text {or }}\right)\right) I\left(\tau_{1}=\tau_{2}\right) .
\end{aligned}
$$

Some examples that are special cases of $\phi\left(u_{1}, u_{2}\right)$ are given as follows. 
1. By letting $w^{\{1\}}(\cdot, \cdot) \equiv w^{\{2\}}(\cdot, \cdot) \equiv w^{\{1,2\}}(\cdot, \cdot) \equiv 1$, the Laplace transform of the time of ruin $T_{\mathrm{or}}$ is retrieved. If one further assumes $\delta=0$, the ruin probability is recovered.

2. If one lets $\delta=0$ and $w^{\{1\}}(\cdot, \cdot) \equiv w^{\{2\}}(\cdot, \cdot) \equiv 0$ and $w^{\{1,2\}}(\cdot, \cdot) \equiv 1$, we obtain the probability that ruin occurs in the sense of $T_{\text {or }}$ with both processes $\left\{U_{1}(t)\right\}_{t \geq 0}$ and $\left\{U_{2}(t)\right\}_{t \geq 0}$ being negative at the ruin time.

3. Assuming $w^{\{1\}}\left(y_{1}, y_{2}\right)=-y_{1}, w^{\{2\}}\left(y_{1}, y_{2}\right)=-y_{2}$ and $w^{\{1,2\}}\left(y_{1}, y_{2}\right)=-y_{1}-y_{2}$, one retrieves the expected discounted deficit at ruin time $T_{\text {or }}$ which only takes into account any negative surplus, namely

$$
E_{\left(u_{1}, u_{2}\right)}\left[e^{-\delta \tau_{1}}\left|U_{1}\left(\tau_{1}\right)\right| I\left(\tau_{1} \leq \tau_{2} ; \tau_{1}<\infty\right)\right]+E_{\left(u_{1}, u_{2}\right)}\left[e^{-\delta \tau_{2}}\left|U_{2}\left(\tau_{2}\right)\right| I\left(\tau_{2} \leq \tau_{1} ; \tau_{2}<\infty\right)\right] .
$$

4. Setting $w^{\{1\}}\left(y_{1}, y_{2}\right)=y_{1}+y_{2}$ and $w^{\{2\}}(\cdot, \cdot) \equiv w^{\{1,2\}}(\cdot, \cdot) \equiv 0$, one obtains the expected discounted total surplus of the two lines of business at time $T_{\text {or }}$ for the case where business 1 is ruined, but business 2 has positive surplus, namely

$$
E_{\left(u_{1}, u_{2}\right)}\left[e^{-\delta \tau_{1}}\left(U_{1}\left(\tau_{1}\right)+U_{2}\left(\tau_{1}\right)\right) I\left(\tau_{1}<\tau_{2}\right)\right] .
$$

Such total surplus is an interesting quantity because if it is positive, this would suggest that it is worth for both lines to continue operation even if line 1 is ruined (see Section 6.2).

In order to apply the recursive methods as in Section 2, we define the Gerber-Shiu function for ruin at the $n$-th claim instant by, for $n=1,2, \ldots$,

$$
\phi_{n}\left(u_{1}, u_{2}, \ldots, u_{M}\right)=E_{\left(u_{1}, u_{2}, \ldots, u_{M}\right)}\left[e^{-\delta T_{\text {or }}} w\left(U_{1}\left(T_{\text {or }}\right), U_{2}\left(T_{\text {or }}\right), \ldots, U_{M}\left(T_{\text {or }}\right)\right) I\left(T_{\text {or }}=S_{n}\right)\right] .
$$

One can then recover the original function in (5) using

$$
\phi\left(u_{1}, u_{2}, \ldots, u_{M}\right)=\sum_{n=1}^{\infty} \phi_{n}\left(u_{1}, u_{2}, \ldots, u_{M}\right)
$$

Our idea here is to evaluate $\phi_{n}\left(u_{1}, u_{2}, \ldots, u_{M}\right)$ recursively in terms of $n$. A starting point would be the quantity $\phi_{1}\left(u_{1}, u_{2}, \ldots, u_{M}\right)$. Due to the representation $(7), \phi_{1}\left(u_{1}, u_{2}, \ldots, u_{M}\right)$ can be represented as the sum of various contributions as

$$
\phi_{1}\left(u_{1}, u_{2}, \ldots, u_{M}\right)=\sum_{i=1}^{M} \phi_{1}^{\{i\}}\left(u_{1}, u_{2}, \ldots, u_{M}\right)+\sum_{A \in \Gamma \backslash \cup_{k=1}^{M}\{k\}} \phi_{1}^{A}\left(u_{1}, u_{2}, \ldots, u_{M}\right),
$$

where for $i=1,2, \ldots, M$,

$$
\begin{aligned}
& \phi_{1}^{\{i\}}\left(u_{1}, u_{2}, \ldots, u_{M}\right) \\
= & E_{\left(u_{1}, u_{2}, \ldots, u_{M}\right)}\left[e^{-\delta S_{1}} w^{\{i\}}\left(U_{1}\left(S_{1}\right), U_{2}\left(S_{1}\right), \ldots, U_{M}\left(S_{1}\right)\right) I\left(\tau_{i}=S_{1} ; \tau_{j}>S_{1} \text { for } j \neq i\right)\right],
\end{aligned}
$$


and for $A \in \Gamma \backslash \cup_{k=1}^{M}\{k\}$,

$$
\begin{aligned}
& \phi_{1}^{A}\left(u_{1}, u_{2}, \ldots, u_{M}\right) \\
= & E_{\left(u_{1}, u_{2}, \ldots, u_{M}\right)}\left[e^{-\delta S_{1}} w^{A}\left(U_{1}\left(S_{1}\right), U_{2}\left(S_{1}\right), \ldots, U_{M}\left(S_{1}\right)\right) I\left(\tau_{i}=S_{1} \text { for } i \in A ; \tau_{j}>S_{1} \text { for } j \notin A\right)\right] .
\end{aligned}
$$

By considering the case where ruin occurs at the first claim instant in the $i$-th line only but not in other lines, a conditional argument leads us to

$$
\begin{aligned}
& \phi_{1}^{\{i\}}\left(u_{1}, \ldots, u_{M}\right) \\
= & \int_{0}^{\infty} \int_{u_{i}+c_{i} t}^{\infty} e^{-\delta t} w^{\{i\}}\left(u_{1}+c_{1} t, \ldots, u_{i-1}+c_{i-1} t, u_{i}+c_{i} t-x_{i}, u_{i+1}+c_{i+1} t, \ldots, u_{M}+c_{M} t\right) f_{i i}\left(x_{1}\right) \lambda_{i i} e^{-\lambda_{s} t} d x_{i} d t \\
& +\int_{0}^{\infty} \int_{0}^{u_{M}+c_{M} t} \cdots \int_{0}^{u_{i+1}+c_{i+1} t} \int_{u_{i}+c_{i} t}^{\infty} \int_{0}^{u_{i-1}+c_{i-1} t} \cdots \int_{0}^{u_{1}+c_{1} t} e^{-\delta t} w^{\{i\}}\left(u_{1}+c_{1} t-x_{1}, \ldots, u_{M}+c_{M} t-x_{M}\right) \\
& \times f_{c}\left(x_{1}, \ldots, x_{M}\right) \lambda_{c} e^{-\lambda_{s} t} d x_{1} \cdots d x_{M} d t \\
= & \int_{0}^{\infty} \int_{0}^{\infty} w^{\{i\}}\left(u_{1}+c_{1} t, \ldots, u_{i-1}+c_{i-1} t,-y_{i}, u_{i+1}+c_{i+1} t, \ldots, u_{M}+c_{M} t\right) f_{i i}\left(u_{i}+c_{i} t+y_{i}\right) \lambda_{i i} e^{-\left(\lambda_{s}+\delta\right) t} d y_{i} d t \\
& +\int_{0}^{\infty} \int_{0}^{u_{M}+c_{M} t} \ldots \int_{0}^{u_{i+1}+c_{i+1} t} \int_{0}^{\infty} \int_{0}^{u_{i-1}+c_{i-1} t} \ldots \int_{0}^{u_{1}+c_{1} t} w^{\{i\}}\left(y_{1}, \ldots, y_{i-1},-y_{i}, y_{i+1} \ldots, y_{M}\right) \\
& \times f_{c}\left(u_{1}+c_{1} t-y_{1}, \ldots, u_{i-1}+c_{i-1} t-y_{i-1}, u_{i}+c_{i} t+y_{i}, u_{i+1}+c_{i+1} t-y_{i+1}, \ldots, u_{M}+c_{M} t-y_{M}\right) \\
& \times \lambda_{c} e^{-\left(\lambda_{s}+\delta\right) t} d y_{1} \cdots d y_{M} d t .
\end{aligned}
$$

Concerning $\phi_{1}^{A}\left(u_{1}, u_{2}, \ldots, u_{M}\right)$ for $A \in \Gamma \backslash \cup_{k=1}^{M}\{k\}$, without loss of generality we may assume we are dealing with a set $A=\left\{i_{1}, i_{2}, \ldots, i_{m}\right\}$ such that $1 \leq i_{1}<i_{2}<\ldots<i_{m} \leq M$ where $2 \leq m \leq M$. Then a similar argument yields

$$
\begin{aligned}
& \phi_{1}^{A}\left(u_{1}, \ldots, u_{M}\right) \\
= & \int_{0}^{\infty} \int_{0}^{u_{M}+c_{M} t} \cdots \int_{0}^{u_{i_{m}+1}+c_{i_{m}+1} t} \int_{u_{i_{m}}+c_{i_{m}} t}^{\infty} \int_{0}^{u_{i_{m}-1}+c_{i_{m}-1} t} \cdots \int_{0}^{u_{i_{1}+1}+c_{i_{1}+1} t} \int_{u_{i_{1}}+c_{i_{1}} t}^{\infty} \int_{0}^{u_{i_{1}-1}+c_{i_{1}-1} t} \\
& \ldots \int_{0}^{u_{1}+c_{1} t} e^{-\delta t} w^{A}\left(u_{1}+c_{1} t-x_{1}, \ldots, u_{M}+c_{M} t-x_{M}\right) f_{c}\left(x_{1}, \ldots, x_{M}\right) \lambda_{c} e^{-\lambda_{s} t} d x_{1} \cdots d x_{M} d t \\
= & \int_{0}^{\infty} \int_{0}^{u_{M}+c_{M} t} \cdots \int_{0}^{u_{i_{m}+1}+c_{i_{m}+1} t} \int_{0}^{\infty} \int_{0}^{u_{i_{m}-1}+c_{i_{m}-1} t} \cdots \int_{0}^{u_{i_{1}+1}+c_{i_{1}+1} t} \int_{0}^{\infty} \int_{i_{1}-1}+c_{i_{1}-1} t \\
& \ldots \int_{0}^{u_{1}+c_{1} t} w^{A}\left(y_{1}, \ldots, y_{i_{1}-1},-y_{i_{1}}, y_{i_{1}+1}, \ldots, y_{i_{m}-1},-y_{i_{m}}, y_{i_{m}+1}, \ldots, y_{M}\right) \\
& \times f_{c}\left(u_{1}+c_{1} t-y_{1}, \ldots, u_{i_{1}-1}+c_{i_{1}-1} t-y_{i_{1}-1}, u_{i_{1}}+c_{i_{1}} t+y_{i_{1}}, u_{i_{1}+1}+c_{i_{1}+1} t-y_{i_{1}+1},\right. \\
& \left.\times u_{i_{m}-1}+c_{i_{m}-1} t-y_{i_{m}-1}, u_{i_{m}}+c_{i_{m}} t+y_{i_{m}}, u_{i_{m}+1}+c_{i_{m}+1} t-y_{i_{m}+1}, \ldots, u_{M}+c_{M} t-y_{M}\right) \\
& \quad \lambda^{-\left(\lambda_{s}+\delta\right) t} d y_{1} \cdots d y_{M} d t .
\end{aligned}
$$

Hence, by utilizing (13) and (14) we obtain the starting point of recursion given by (12). In general, for the $(n+1)$-th step, we condition on the time and amount of the first claim without 
ruin occurring to obtain the recursive relationship (which is analogous to (3)) for $n=1,2, \ldots$,

$$
\begin{aligned}
& \phi_{n+1}\left(u_{1}, \ldots, u_{M}\right) \\
= & \sum_{i=1}^{M} \int_{0}^{\infty} \int_{0}^{u_{i}+c_{i} t} \phi_{n}\left(u_{1}+c_{1} t, \ldots, u_{i-1}+c_{i-1} t, u_{i}+c_{i} t-x_{i}, u_{i+1}+c_{i+1} t, \ldots, u_{M}+c_{M} t\right) f_{i i}\left(x_{1}\right) \lambda_{i i} e^{-\left(\lambda_{s}+\delta\right) t} d x_{i} d t \\
& +\int_{0}^{\infty} \int_{0}^{u_{M}+c_{M} t} \cdots \int_{0}^{u_{1}+c_{1} t} \phi_{n}\left(u_{1}+c_{1} t-x_{1}, \ldots, u_{M}+c_{M} t-x_{M}\right) f_{c}\left(x_{1}, \ldots, x_{M}\right) \\
& \times \lambda_{c} e^{-\left(\lambda_{s}+\delta\right) t} d x_{1} \cdots d x_{M} d t .
\end{aligned}
$$

Example 4 In the bivariate version of model (2), the starting point of the recursion (12) consists of three contributions, i.e.

$$
\phi_{1}\left(u_{1}, u_{2}\right)=\phi_{1}^{\{1\}}\left(u_{1}, u_{2}\right)+\phi_{1}^{\{2\}}\left(u_{1}, u_{2}\right)+\phi_{1}^{\{1,2\}}\left(u_{1}, u_{2}\right),
$$

where $\phi_{1}^{\{1\}}\left(u_{1}, u_{2}\right), \phi_{1}^{\{2\}}\left(u_{1}, u_{2}\right)$ and $\phi_{1}^{\{1,2\}}\left(u_{1}, u_{2}\right)$ correspond to the cases $\left\{\tau_{1}<\tau_{2}\right\},\left\{\tau_{2}<\tau_{1}\right\}$ and $\left\{\tau_{1}=\tau_{2}\right\}$ respectively. In some cases, the three contributions simplify. For instance, if one is interested in the expected discounted deficit at ruin time $T_{\text {or }}$ defined in (8) under the penalty functions $w^{\{1\}}\left(y_{1}, y_{2}\right)=-y_{1}, w^{\{2\}}\left(y_{1}, y_{2}\right)=-y_{2}$ and $w^{\{1,2\}}\left(y_{1}, y_{2}\right)=-y_{1}-y_{2}$, one has that

$$
\begin{aligned}
\phi_{1}^{\{1\}}\left(u_{1}, u_{2}\right) & =\int_{0}^{\infty} \int_{0}^{\infty} y f_{1}\left(u_{1}+c_{1} t+y\right)\left[\lambda_{11}+\lambda_{12} F_{2}\left(u_{2}+c_{2} t\right)\right] e^{-\left(\lambda_{s}+\delta\right) t} d y d t \\
\phi_{1}^{\{2\}}\left(u_{1}, u_{2}\right) & =\int_{0}^{\infty} \int_{0}^{\infty} y f_{2}\left(u_{2}+c_{2} t+y\right)\left[\lambda_{22}+\lambda_{12} F_{1}\left(u_{1}+c_{1} t\right)\right] e^{-\left(\lambda_{s}+\delta\right) t} d y d t \\
\phi_{1}^{\{1,2\}}\left(u_{1}, u_{2}\right) & =\int_{0}^{\infty} \int_{0}^{\infty} y \int_{u_{1}+c_{1} t}^{u_{1}+c_{1} t+y} f_{1}\left(x_{1}\right) f_{2}\left(u_{2}+c_{2} t+u_{1}+c_{1} t+y-x_{1}\right) \lambda_{12} e^{-\left(\lambda_{s}+\delta\right) t} d x_{1} d y d t
\end{aligned}
$$

where $F_{i}(\cdot)$ represents the cumulative distribution function corresponding to the density $f_{i}(\cdot)$ for $i=1,2$.

\section{Reduction of recursive integrals to recursive sums}

As mentioned at the end of Section 2, a direct application of the recursive integrals in the form of (3) requires long execution times especially due to the fact that we aim at letting $n \rightarrow \infty$, and the algorithm may be numerically instable as well. The same comments also apply to the computation of the Gerber-Shiu function (10) for ruin at the $n$-th claim via (15). Inspired by Stanford and Stroiński (1994) and Stanford et al. (2000) (who worked with the univariate risk process), in the next two subsections we study two examples in which the recursive integration previously presented can be explicitly evaluated when distributional assumptions on the claims are made. More precisely, recursive integrals can be reduced to give recursive sums, therefore providing a computational tractable solution. For simplicity, in this entire section we will consider a bivariate version of the model (2). 


\subsection{Mixture of Erlangs claims - Survival probability}

In order to show the general applicability of the recursive methods with regards to the choice of claim size distributions, we assume that the claims in the both lines are mixture of Erlangs with common scale parameter, with densities given by $f_{1}\left(x_{1}\right)=\sum_{i=1}^{m} q_{i} \beta_{1}^{i} x_{1}^{i-1} e^{-\beta_{1} x_{1}} /(i-1)$ ! and $f_{2}\left(x_{2}\right)=\sum_{j=1}^{m} p_{j} \beta_{2}^{j} x_{2}^{j-1} e^{-\beta_{2} x_{2}} /(j-1)$ ! respectively. The class of mixed Erlang distributions is very flexible and versatile being an ideal candidate to fit insurance data (see Willmot and Woo (2007) and Lee and Lin (2010) for an exhaustive analysis). Due to increased complexity in calculations, in this subsection we restrict ourselves to deriving explicit recursive expressions for the survival probabilities studied in Section 2 only in the common shock case, i.e. $\lambda_{11}=\lambda_{22}=0$ and $\lambda_{c}=\lambda_{12}>0$.

The idea that we use to find the general solution of (3) is based on mathematical induction. We anticipate the form of the solution at the $n$-th step, assume it correct, and prove it at the $(n+1)$-th step. Thus, we obtain recursive formulas for the coefficients at the $(n+1)$-th step in terms of the coefficients at the previous $n$-th step. To begin, using equation (3) for $n=0$ and $\lambda_{11}=\lambda_{22}=0$ along with the trivial condition $\Phi_{0}\left(u_{1}, u_{2}\right)=1$, after some tedious but rather simple calculations we obtain

$$
\begin{aligned}
\Phi_{1}\left(u_{1}, u_{2}\right) & =\int_{0}^{\infty} \int_{0}^{u_{2}+c_{2} t} \int_{0}^{u_{1}+c_{1} t} f_{1}\left(x_{1}\right) f_{2}\left(x_{2}\right) \lambda_{12} e^{-\lambda_{12} t} d x_{1} d x_{2} d t \\
& =1-\sum_{s=0}^{m-1} a_{[1, s]} u_{1}^{s} e^{-\beta_{1} u_{1}}-\sum_{s=0}^{m-1} b_{[1, s]} u_{2}^{s} e^{-\beta_{2} u_{2}}+\sum_{s=0}^{m-1} \sum_{v=0}^{m-1} e_{[1, s, v]} u_{1}^{s} u_{2}^{v} e^{-\left(\beta_{1} u_{1}+\beta_{2} u_{2}\right)},
\end{aligned}
$$

where

$$
\begin{aligned}
a_{[1, s]} & =\sum_{i=s+1}^{m} \sum_{k=s}^{i-1} \frac{\lambda_{12} q_{i} c_{1}^{k-s} \beta_{1}^{k}}{s !(k-s) !\left(\lambda_{12}+c_{1} \beta_{1}\right)^{k-s+1}}, \\
b_{[1, s]} & =\sum_{j=s+1}^{m} \sum_{k=s}^{j-1} \frac{\lambda_{12} p_{j} c_{2}^{k-s} \beta_{2}^{k}}{s !(k-s) !\left(\lambda_{12}+c_{2} \beta_{2}\right)^{k-s+1}}, \\
e_{[1, s, v]} & =\sum_{i=s+1}^{m} \sum_{k=s}^{i-1} \sum_{j=v+1}^{m} \sum_{l=v}^{j-1}\left(\begin{array}{c}
k+l-s-v \\
k-s
\end{array}\right) \frac{\lambda_{12} p_{j} q_{i} c_{1}^{k-s} c_{2}^{l-v} \beta_{1}^{k} \beta_{2}^{l}}{s ! v ! k ! l !\left(\lambda_{12}+c_{1} \beta_{1}+c_{2} \beta_{2}\right)^{k+l-s-v+1}}
\end{aligned}
$$

for $s, v=0,1, \ldots, m-1$. Continuing further, we observe that the general form of the solution at the $n$-th step is given by, for $n=1,2, \ldots$,

$\Phi_{n}\left(u_{1}, u_{2}\right)=1-\sum_{w=0}^{n m-1} a_{[n, w]} u_{1}^{w} e^{-\beta_{1} u_{1}}-\sum_{w=0}^{n m-1} b_{[n, w]} u_{2}^{w} e^{-\beta_{2} u_{2}}+\sum_{w=0}^{n m-1} \sum_{y=0}^{n m-1} e_{[n, w, y]} u_{1}^{w} u_{2}^{y} e^{-\left(\beta_{1} u_{1}+\beta_{2} u_{2}\right)}$.

Here $a_{[\cdot, \cdot]}, b_{[\cdot, \cdot]}, e_{[\cdot, \cdot, \cdot]}$ are constant coefficients (i.e. independent of $u_{1}$ and $u_{2}$ ) that will be evaluated recursively in two or three dimensions. See also Remark 2. Assuming that (22) holds for some $n \geq 1$ and plugging it into (3), after some tedious calculations whose details are omitted here, it can be shown that (22) holds true with $n+1$ in place of $n$, where the coefficients $a_{[n+1, \cdot]}, b_{[n+1, \cdot]}$, $e_{[n+1, \cdot, \cdot]}$ at the $(n+1)$-th step are given recursively in the following Proposition. 
Proposition 1 The survival probability can be calculated as $\Phi\left(u_{1}, u_{2}\right)=\lim _{n \rightarrow \infty} \Phi_{n}\left(u_{1}, u_{2}\right)$ where the general term is given by

$$
\begin{aligned}
\Phi_{n+1}\left(u_{1}, u_{2}\right)= & 1-\sum_{w=0}^{(n+1) m-1} a_{[n+1, w]} u_{1}^{w} e^{-\beta_{1} u_{1}}-\sum_{w=0}^{(n+1) m-1} b_{[n+1, w]} u_{2}^{w} e^{-\beta_{2} u_{2}} \\
& +\sum_{w=0}^{(n+1) m-1} \sum_{y=0}^{(n+1) m-1} e_{[n+1, w, y]} u_{1}^{w} u_{2}^{y} e^{-\left(\beta_{1} u_{1}+\beta_{2} u_{2}\right)},
\end{aligned}
$$

for $n=0,1, \ldots$, with

$$
\begin{aligned}
& a_{[n+1, w]}=a_{[1, w]} I(0 \leq w \leq m-1) \\
& +\sum_{i=\max (1, w-m n+1)}^{m} \sum_{s=\max (0, w-i)}^{n m-1} \sum_{g=0}^{s} \lambda_{12} q_{i} a_{[n, s]} \frac{(-1)^{s-g} c_{1}^{s+i-w} \beta_{1}^{i}(s+i) ! s !}{(i-1) ! g !(s-g) ! w !(s-g+i)\left(\lambda_{12}+c_{1} \beta_{1}\right)^{s+i+1-w}}, \\
& b_{[n+1, w]}=b_{[1, w]} I(0 \leq w \leq m-1) \\
& +\sum_{j=\max (1, w-m n+1)}^{m} \sum_{s=\max (0, w-j)}^{n m-1} \sum_{g=0}^{s} \lambda_{12} p_{j} b_{[n, s]} \frac{(-1)^{s-g} c_{2}^{s+j-w} \beta_{2}^{j}(s+j) ! s !}{(j-1) ! g !(s-g) ! w !(s-g+j)\left(\lambda_{12}+c_{2} \beta_{2}\right)^{s+j+1-w}}, \\
& e_{[n+1, w, y]}=e_{[1, w, y]} I(0 \leq w \leq m-1,0 \leq y \leq m-1) \\
& +I(0 \leq y \leq m-1) \sum_{i=\max (1, w-m n+1)}^{m} \sum_{s=\max (0, w-i)}^{n m-1} \sum_{g=0}^{s} \sum_{j=y+1}^{m} \sum_{l=y}^{j-1} \lambda_{12} p_{j} q_{i} a_{[n, s]} \\
& \times\left(\begin{array}{c}
s+i+l-w-y \\
s+i-w
\end{array}\right)\left(\begin{array}{c}
s \\
s-g
\end{array}\right) \frac{(-1)^{s-g} c_{1}^{s+i-w} c_{2}^{l-y} \beta_{1}^{i} \beta_{2}^{l}(s+i) !}{(i-1) ! w ! y !(s-g+i)\left(\lambda_{12}+c_{1} \beta_{1}+c_{2} \beta_{2}\right)^{s+i+l-w-y+1}} \\
& +I(0 \leq w \leq m-1) \sum_{j=\max (1, y-m n+1)}^{m} \sum_{s=\max (0, y-j)}^{n m-1} \sum_{g=0}^{s} \sum_{i=w+1}^{m} \sum_{k=w}^{i-1} \lambda_{12} p_{j} q_{i} b_{[n, s]} \\
& \times\left(\begin{array}{c}
s+j+k-w-y \\
s+j-y
\end{array}\right)\left(\begin{array}{c}
s \\
s-g
\end{array}\right) \frac{(-1)^{s-g} c_{1}^{k-w} c_{2}^{s+j-y} \beta_{1}^{k} \beta_{2}^{j}(s+j) !}{(j-1) ! w ! y !(s-g+j)\left(\lambda_{12}+c_{1} \beta_{1}+c_{2} \beta_{2}\right)^{k+j+s-w-y+1}} \\
& +\sum_{i=\max (1, w-m n+1)}^{m} \sum_{s=\max (0, w-i)}^{n m-1} \sum_{g=0}^{s} \sum_{j=\max (1, y-m n+1)}^{m} \sum_{v=\max (0, y-j)}^{n m-1} \sum_{z=0}^{v} \frac{\lambda_{12} p_{j} q_{i} e_{[n, s, v]}}{(s+i-g)(v+j-z)} \\
& \times\left(\begin{array}{c}
s+i+v+j-w-y \\
s+i-w
\end{array}\right)\left(\begin{array}{c}
s \\
s-g
\end{array}\right)\left(\begin{array}{c}
v \\
v-z
\end{array}\right) \frac{(-1)^{s+v-g-z} c_{1}^{s+i-w} c_{2}^{v+j-y} \beta_{1}^{i} \beta_{2}^{j}(s+i) !(v+j) !}{(i-1) !(j-1) ! w ! y !\left(\lambda_{12}+c_{1} \beta_{1}+c_{2} \beta_{2}\right)^{s+v+i+j-w-y+1}},
\end{aligned}
$$

for $n=1,2, \ldots ; w, y=0,1, \ldots,(n+1) m-1$. The starting point of the recursion is given by (19), (20) and (21).

\subsection{Exponential claims - Expected discounted deficit}

In this subsection, we assume exponential claims with densities $f_{i}\left(x_{i}\right)=\mu_{i} e^{-\mu_{i} x_{i}}$ for $i=1,2$, in the bivariate risk process defined in (2), and we assume $w^{\{1\}}\left(y_{1}, y_{2}\right)=-y_{1}, w^{\{2\}}\left(y_{1}, y_{2}\right)=-y_{2}$ 
and $w^{\{1,2\}}\left(y_{1}, y_{2}\right)=-y_{1}-y_{2}$ so that we find the quantity (8) (see Example 4). Starting at $n=1$, after simple manipulations (16), (17) and (18) become

$$
\begin{aligned}
\phi_{1}^{\{1\}}\left(u_{1}, u_{2}\right) & =\frac{\lambda_{11}+\lambda_{12}}{\mu_{1}\left(\lambda_{s}+\delta+c_{1} \mu_{1}\right)} e^{-\mu_{1} u_{1}}-\frac{\lambda_{12}}{\mu_{1}\left(\lambda_{s}+\delta+c_{1} \mu_{1}+c_{2} \mu_{2}\right)} e^{-\left(\mu_{1} u_{1}+\mu_{2} u_{2}\right)}, \\
\phi_{1}^{\{2\}}\left(u_{1}, u_{2}\right) & =\frac{\lambda_{22}+\lambda_{12}}{\mu_{2}\left(\lambda_{s}+\delta+c_{2} \mu_{2}\right)} e^{-\mu_{2} u_{2}}-\frac{\lambda_{12}}{\mu_{2}\left(\lambda_{s}+\delta+c_{1} \mu_{1}+c_{2} \mu_{2}\right)} e^{-\left(\mu_{1} u_{1}+\mu_{2} u_{2}\right)}, \\
\phi_{1}^{\{1,2\}}\left(u_{1}, u_{2}\right) & =\left(\frac{1}{\mu_{1}}+\frac{1}{\mu_{2}}\right) \frac{\lambda_{12}}{\lambda_{s}+\delta+c_{1} \mu_{1}+c_{2} \mu_{2}} e^{-\left(\mu_{1} u_{1}+\mu_{2} u_{2}\right)} .
\end{aligned}
$$

Therefore, the expected discounted deficit when ruin happens at the instant of the first claim is given by

$$
\phi_{1}\left(u_{1}, u_{2}\right)=\frac{\lambda_{11}+\lambda_{12}}{\mu_{1}\left(\lambda_{s}+\delta+c_{1} \mu_{1}\right)} e^{-\mu_{1} u_{1}}+\frac{\lambda_{22}+\lambda_{12}}{\mu_{2}\left(\lambda_{s}+\delta+c_{2} \mu_{2}\right)} e^{-\mu_{2} u_{2}} .
$$

With the above formula serving as the starting point, applying the same procedure as in the previous subsection, one obtains the following Proposition by mathematical induction.

Proposition 2 The expected discounted deficit at ruin can be calculated as $\phi\left(u_{1}, u_{2}\right)=\sum_{n=1}^{\infty} \phi_{n}\left(u_{1}, u_{2}\right)$ where the general term is given by

$$
\phi_{n+1}\left(u_{1}, u_{2}\right)=\sum_{j=0}^{n} a_{[n+1, j]} u_{1}^{j} e^{-\mu_{1} u_{1}}+\sum_{j=0}^{n} b_{[n+1, j]} u_{2}^{j} e^{-\mu_{2} u_{2}}-\sum_{j=0}^{n} \sum_{k=0}^{n} e_{[n+1, j, k]} u_{1}^{j} u_{2}^{k} e^{-\left(\mu_{1} u_{1}+\mu_{2} u_{2}\right)},
$$

for $n=0,1, \ldots$, with

$$
\begin{aligned}
a_{[n+1, j]}= & \sum_{i=\max (j-1,0)}^{n-1} \frac{a_{[1,0]} \mu_{1}^{2} a_{[n, i]} i ! c_{1}^{i+1-j}}{j !\left(\lambda_{s}+\delta+c_{1} \mu_{1}\right)^{i+1-j}}+\sum_{i=j}^{n-1} \frac{\lambda_{22} a_{[n, i]} i ! c_{1}^{i-j}}{j !\left(\lambda_{s}+\delta+c_{1} \mu_{1}\right)^{i+1-j}}, \\
b_{[n+1, j]}= & \sum_{i=\max (j-1,0)}^{n-1} \frac{b_{[1,0]} \mu_{2}^{2} b_{[n, i]} i ! c_{2}^{i+1-j}}{j !\left(\lambda_{s}+\delta+c_{2} \mu_{2}\right)^{i+1-j}}+\sum_{i=j}^{n-1} \frac{\lambda_{11} b_{[n, i]} i ! c_{2}^{i-j}}{j !\left(\lambda_{s}+\delta+c_{2} \mu_{2}\right)^{i+1-j}}, \\
e_{[n+1, j, k]}= & I(k=0)\left(\sum_{i=\max (0, j-1)}^{n-1} \frac{\rho \mu_{1} a_{[n, i]} c_{1}^{i+1-j} i !}{j !\left(\lambda_{s}+\delta+c_{1} \mu_{1}+c_{2} \mu_{2}\right)^{i+1-j}}+\sum_{i=j}^{n-1} \frac{\lambda_{22} a_{[n, i]} c_{1}^{i-j} i !}{j !\left(\lambda_{s}+\delta+c_{1} \mu_{1}+c_{2} \mu_{2}\right)^{i+1-j}}\right) \\
& +I(j=0)\left(\sum_{i=\max (0, k-1)}^{n-1} \frac{{ }^{i+1} \mu_{2} b_{[n, i]} c_{2}^{i+1-k} i !}{k !\left(\lambda_{s}+\delta+c_{1} \mu_{1}+c_{2} \mu_{2}\right)^{i+1-k}+\sum_{i=j} \frac{\lambda_{11} b_{[n, i]} c_{2}^{i-k} i !}{k !\left(\lambda_{s}+\delta+c_{1} \mu_{1}+c_{2} \mu_{2}\right)^{i+1-k}}}\right) \\
& +\sum_{i=j}^{n-1} \sum_{q=\max (0, k-1)}^{n-1}\left(\begin{array}{c}
i+q+1-j-k \\
i-j
\end{array}\right) \frac{e_{[n, i, q]} \lambda_{22} \mu_{2} i ! q ! c_{1}^{i-j} c_{2}^{q+1-k}}{j ! k !\left(\lambda_{s}+\delta+c_{1} \mu_{1}+c_{2} \mu_{2}\right)^{i+q+2-j-k}} \\
& +\sum_{q=k}^{n-1} \sum_{i=\max (0, j-1)}^{n-1}\left(\begin{array}{c}
i+q+1-j-k \\
q-k
\end{array}\right) \frac{e_{[n, i, q]} \lambda_{11} \mu_{1} i ! q ! c_{1}^{i+1-j} c_{2}^{q-k}}{j ! k !\left(\lambda_{s}+\delta+c_{1} \mu_{1}+c_{2} \mu_{2}\right)^{i+q+2-j-k}} \\
& +\sum_{q=\max (0, k-1)}^{n-1} \sum_{i=\max (0, j-1)}^{n-1}\left(\begin{array}{c}
i+q+2-j-k \\
q+1-k
\end{array}\right) \frac{e_{[n, i, q]} \lambda_{12} \mu_{1} \mu_{2} i ! q ! c_{1}^{i+1-j} c_{2}^{q+1-k}}{j ! k !\left(\lambda_{s}+\delta+c_{1} \mu_{1}+c_{2} \mu_{2}\right)^{i+q+3-j-k}},
\end{aligned}
$$


for $n=1,2, \ldots ; j, k=0,1, \ldots, n$, where $\rho=\lambda_{12} /\left(\lambda_{s}+\delta+c_{1} \mu_{1}+c_{2} \mu_{2}\right)$. The starting point of the recursion is given by

$$
a_{[1,0]}=\frac{\lambda_{11}+\lambda_{12}}{\mu_{1}\left(\lambda_{s}+\delta+c_{1} \mu_{1}\right)}, \quad b_{[1,0]}=\frac{\lambda_{22}+\lambda_{12}}{\mu_{2}\left(\lambda_{s}+\delta+c_{2} \mu_{2}\right)}, \quad e_{[1,0,0]}=0 .
$$

As in the previous subsection, we avoid to present the detailed proof on Proposition 2 due to the tedious algebraic calculations involved.

Remark 2 Despite the rather messy appearance of the formulas in Propositions 1 and 2, the results provide computationally more tractable solutions for the survival probability and the expected discounted deficit at ruin, since we only need to calculate recursive sums instead of recursive integrals. It is also instructive to note that in both Propositions 1 and 2 , the coefficients $a_{[\cdot, \cdot]}, b_{[\cdot, \cdot]}$, $e_{[\cdot,, \cdot]}$ do not depend on $\left(u_{1}, u_{2}\right)$. In other words, once the coefficients (up to the $n$-th step) have been determined, the same ruin-related quantity under any combinations of initial surplus levels $\left(u_{1}, u_{2}\right)$ follows immediately. This is in contrast to the recursive integral formulas (3) and (15) in which a separate numerical integration has to be performed for a different set of $\left(u_{1}, u_{2}\right)$. Such an appealing feature of the method of recursive sums presented here makes it relatively easy to study various optimal allocation problems, for example, in minimizing the joint ruin probability (see Section 6.3) or the expected discounted deficit.

\section{$5 \quad$ Survival probability for $T_{\text {and }}$}

In this section, we turn our attention to the survival probability associated to the time of ruin $T_{\text {and }}=\max \left(\tau_{1}, \tau_{2}\right)$ under the framework (1) in the bivariate case (see Remark 4 for discussion about higher dimensions). Such survival probability will be denoted by

$$
\Phi^{\text {and }}\left(u_{1}, u_{2}\right)=\operatorname{Pr}{ }_{\left(u_{1}, u_{2}\right)}\left(T_{\text {and }}=\infty\right)=\operatorname{Pr}\left(u_{1}, u_{2}\right)\left(\tau_{1}=\infty \text { or } \tau_{2}=\infty\right),
$$

and hence $\Psi^{\text {and }}\left(u_{1}, u_{2}\right)=1-\Phi^{\text {and }}\left(u_{1}, u_{2}\right)$ represents the associated ruin probability. By recalling that $S_{n}$ is the time of the $n$-th claim event, in parallel to the beginning of Section 2 , for $n=0,1, \ldots$ we define the survival probability

$$
\Phi_{n}^{\text {and }}\left(u_{1}, u_{2}\right)=1-\Psi_{n}^{\text {and }}\left(u_{1}, u_{2}\right)=\operatorname{Pr}{ }_{\left(u_{1}, u_{2}\right)}\left(T_{\text {and }}>S_{n}\right),
$$

as well as the univariate survival probabilities, for $i=1,2$,

$$
\Phi_{n}^{i}\left(u_{i}\right)=1-\Psi_{n}^{i}\left(u_{i}\right)=\operatorname{Pr}\left(\tau_{i}>S_{n} \mid U_{i}(0)=u_{i}\right) .
$$

If we define the sets $A_{i}=\left\{\tau_{i}>S_{n}\right\}$ for $i=1,2$, then $\left\{T_{\text {and }}>S_{n}\right\}=\left\{\max \left(\tau_{1}, \tau_{2}\right)>S_{n}\right\}=A_{1} \cup A_{2}$ and $\left\{T_{\text {or }}>S_{n}\right\}=\left\{\min \left(\tau_{1}, \tau_{2}\right)>S_{n}\right\}=A_{1} \cap A_{2}$. Using the identity $\operatorname{Pr}\left(A_{1} \cup A_{2}\right)=\operatorname{Pr}\left(A_{1}\right)+$ $\operatorname{Pr}\left(A_{2}\right)-\operatorname{Pr}\left(A_{1} \cap A_{2}\right)$, one immediately has that, for $n=0,1, \ldots$,

$$
\Phi_{n}^{\text {and }}\left(u_{1}, u_{2}\right)=\Phi_{n}^{1}\left(u_{1}\right)+\Phi_{n}^{2}\left(u_{2}\right)-\Phi_{n}\left(u_{1}, u_{2}\right) .
$$

The implication of (24) is that, if we can determine $\Phi_{n}^{i}\left(u_{i}\right)$ for $i=1,2$ then $\Phi_{n}^{\text {and }}\left(u_{1}, u_{2}\right)$ follows accordingly with $\Phi_{n}\left(u_{1}, u_{2}\right)$ obtained from earlier sections. Then the ultimate survival probability is given by $\Phi^{\text {and }}\left(u_{1}, u_{2}\right)=\lim _{n \rightarrow \infty} \Phi_{n}^{\text {and }}\left(u_{1}, u_{2}\right)$. 
Remark 3 Note that according to the definition (23), a claim event which generates a claim in line 1 but not in line 2 should also be counted as a 'claim event' in line 2, and vice versa. This is particularly important for a fair comparison between the bivariate and univariate probabilities as well as ensuring the relationship (24) holds. Therefore, the way the number of claims is counted here is different from that in Stanford and Stroinski (1994) and Stanford et al. (2000). Note also that $\Phi_{n}^{1}\left(u_{1}\right)$ is independent of $u_{2}, c_{2}$ and $f_{2}(\cdot)$ but dependent on $\lambda_{22}$ pertaining to the process $\left\{U_{2}(t)\right\}_{t \geq 0}$. Similar comments apply to $\Phi_{n}^{2}\left(u_{2}\right)$.

Given (24), it remains to derive recursive relationships for the univariate survival probabilities $\Phi_{n}^{i}\left(u_{i}\right)$ for $i=1,2$. Since we denote $\lambda_{c}=\lambda_{12}$ for bivariate process, we shall also write $f_{c}(\cdot, \cdot) \equiv$ $f_{12}(\cdot, \cdot)$. When $n=1$, by conditioning on the time and claim amounts upon the arrival of the first claim event we obtain

$$
\begin{aligned}
\Phi_{1}^{1}\left(u_{1}\right)= & \int_{0}^{\infty} \int_{0}^{u_{1}+c_{1} t} f_{11}\left(x_{1}\right) \lambda_{11} e^{-\lambda_{s} t} d x_{1} d t+\int_{0}^{\infty} \int_{0}^{\infty} f_{22}\left(x_{2}\right) \lambda_{22} e^{-\lambda_{s} t} d x_{2} d t \\
& +\int_{0}^{\infty} \int_{0}^{\infty} \int_{0}^{u_{1}+c_{1} t} f_{12}\left(x_{1}, x_{2}\right) \lambda_{12} e^{-\lambda_{s} t} d x_{1} d x_{2} d t \\
= & \int_{0}^{\infty}\left[F_{11}\left(u_{1}+c_{1} t\right) \lambda_{11}+F_{1} \bullet\left(u_{1}+c_{1} t\right) \lambda_{12}\right] e^{-\lambda_{s} t} d t+\frac{\lambda_{22}}{\lambda_{s}} \\
= & 1-\int_{0}^{\infty}\left[\bar{F}_{11}\left(u_{1}+c_{1} t\right) \lambda_{11}+\bar{F}_{1} \bullet\left(u_{1}+c_{1} t\right) \lambda_{12}\right] e^{-\lambda_{s} t} d t,
\end{aligned}
$$

where $F_{11}(\cdot)=1-\bar{F}_{11}(\cdot)$ and $F_{1} \bullet(\cdot)=1-\bar{F}_{1} \bullet(\cdot)$ represent the cumulative distribution functions corresponding to the densities $f_{11}(\cdot)$ and $\int_{0}^{\infty} f_{12}\left(\cdot, x_{2}\right) d x_{2}$ respectively. Recursively, one has that for $n=0,1, \ldots$,

$$
\begin{aligned}
\Phi_{n+1}^{1}\left(u_{1}\right)= & \int_{0}^{\infty} \int_{0}^{u_{1}+c_{1} t} \Phi_{n}^{1}\left(u_{1}+c_{1} t-x_{1}\right)\left\{f_{11}\left(x_{1}\right) \lambda_{11}+\left[\int_{0}^{\infty} f_{12}\left(x_{1}, x_{2}\right) d x_{2}\right] \lambda_{12}\right\} e^{-\lambda_{s} t} d x_{1} d t \\
& +\int_{0}^{\infty} \Phi_{n}^{1}\left(u_{1}+c_{1} t\right) \lambda_{22} e^{-\lambda_{s} t} d t .
\end{aligned}
$$

The study of $\Phi_{n}^{2}\left(u_{2}\right)$ can be done in an identical manner by reversing the roles of lines 1 and 2 .

The following example demonstrates how the same ideas as in Section 4 can be employed to replace the recursive integrals in (25) by recursive sums using mathematical induction.

Example 5 As in Example 1, we consider the bivariate version of model (2) under exponential assumptions with $f_{i}\left(x_{i}\right)=\mu_{i} e^{-\mu_{i} x_{i}}$ for $i=1,2$. Omitting the straightforward algebraic details, line 1 's survival probability $\Phi_{n+1}^{1}\left(u_{1}\right)$ up to and including the $(n+1)$-th claim events admits the representation

$$
\Phi_{n+1}^{1}\left(u_{1}\right)=1-\sum_{j=0}^{n} a_{[n+1, j]}^{1} u_{1}^{j} e^{-\mu_{1} u_{1}}
$$

for $n=0,1, \ldots$, with

$$
a_{[n+1, j]}^{1}=a_{[1,0]}^{1} I(j=0)+\sum_{i=\max (j-1,0)}^{n-1} \frac{a_{[n, i]}^{1}\left(\lambda_{11}+\lambda_{12}\right) \mu_{1} c_{1}^{i+1-j} i !}{j !\left(\lambda_{s}+c_{1} \mu_{1}\right)^{i+2-j}}+\sum_{i=j}^{n-1} \frac{a_{[n, i]}^{1} \lambda_{22} c_{1}^{i-j} i !}{j !\left(\lambda_{s}+c_{1} \mu_{1}\right)^{i+1-j}},
$$

for $n=1,2, \ldots ; j=0,1, \ldots, n$. The starting point is $a_{[1,0]}^{1}=\left(\lambda_{11}+\lambda_{12}\right) /\left(\lambda_{s}+c_{1} \mu_{1}\right)$. 
Remark 4 The survival probability for $T_{\text {and }}$ in the general multi-dimensional case can in principle be studied in the same manner using the inclusion-exclusion principle. To illustrate this, we briefly discuss the trivariate case (i.e. $M=3$ ) as follows. We are interested in computing the quantity $\Phi_{n}^{\text {and }}\left(u_{1}, u_{2}, u_{3}\right)=\operatorname{Pr}{ }_{\left(u_{1}, u_{2}, u_{3}\right)}\left(T_{\text {and }}>S_{n}\right)$ so that

$$
\Phi^{\text {and }}\left(u_{1}, u_{2}, u_{3}\right)=\operatorname{Pr}\left(u_{1}, u_{2}, u_{3}\right)\left(T_{\text {and }}=\infty\right)=\lim _{n \rightarrow \infty} \Phi_{n}^{\text {and }}\left(u_{1}, u_{2}, u_{3}\right) .
$$

While the univariate survival probabilities $\Phi_{n}^{i}\left(u_{i}\right)$ for $i=1,2,3$ have the same representation as in (23) (with the understanding that $S_{n}$ is defined in the trivariate framework), we further define the bivariate survival probabilities, for $i, j \in\{1,2,3\}$ and $i<j$,

$$
\Phi_{n}^{i j}\left(u_{i}, u_{j}\right)=\operatorname{Pr}\left(\tau_{i}>S_{n} \text { and } \tau_{j}>S_{n} \mid\left(U_{i}(0), U_{j}(0)\right)=\left(u_{i}, u_{j}\right)\right) .
$$

If $A_{i}=\left\{\tau_{i}>S_{n}\right\}$ for $i=1,2,3$, then $\left\{T_{\text {and }}>S_{n}\right\}=A_{1} \cup A_{2} \cup A_{3}$ and $\left\{T_{\text {or }}>S_{n}\right\}=A_{1} \cap A_{2} \cap A_{3}$, and therefore with the identity $\operatorname{Pr}\left(A_{1} \cup A_{2} \cup A_{3}\right)=\operatorname{Pr}\left(A_{1}\right)+\operatorname{Pr}\left(A_{2}\right)+\operatorname{Pr}\left(A_{3}\right)-\operatorname{Pr}\left(A_{1} \cap A_{2}\right)-$ $\operatorname{Pr}\left(A_{1} \cap A_{3}\right)-\operatorname{Pr}\left(A_{2} \cap A_{3}\right)+\operatorname{Pr}\left(A_{1} \cap A_{2} \cap A_{3}\right)$ we immediately arrive at, for $n=0,1, \ldots$,

$\Phi_{n}^{\text {and }}\left(u_{1}, u_{2}, u_{3}\right)=\Phi_{n}^{1}\left(u_{1}\right)+\Phi_{n}^{2}\left(u_{2}\right)+\Phi_{n}^{2}\left(u_{2}\right)-\Phi_{n}^{12}\left(u_{1}, u_{2}\right)-\Phi_{n}^{13}\left(u_{1}, u_{3}\right)-\Phi_{n}^{23}\left(u_{2}, u_{3}\right)+\Phi_{n}\left(u_{1}, u_{2}, u_{3}\right)$.

The univariate survival probability $\Phi_{n}^{1}\left(u_{1}\right)$ satisfies the recursion, for $n=0,1, \ldots$,

$$
\begin{aligned}
\Phi_{n+1}^{1}\left(u_{1}\right)= & \int_{0}^{\infty} \int_{0}^{u_{1}+c_{1} t} \Phi_{n}^{1}\left(u_{1}+c_{1} t-x_{1}\right)\left\{f_{11}\left(x_{1}\right) \lambda_{11}+\left[\int_{0}^{\infty} \int_{0}^{\infty} f_{c}\left(x_{1}, x_{2}, x_{3}\right) d x_{2} d x_{3}\right] \lambda_{c}\right\} e^{-\lambda_{s} t} d x_{1} d t \\
& +\int_{0}^{\infty} \Phi_{n}^{1}\left(u_{1}+c_{1} t\right)\left(\lambda_{22}+\lambda_{33}\right) e^{-\lambda_{s} t} d t
\end{aligned}
$$

with starting value $\Phi_{0}^{1}\left(u_{1}\right)=1$. Similarly, the bivariate survival probability $\Phi_{n}^{12}\left(u_{1}, u_{2}\right)$ is given by, for $n=0,1, \ldots$,

$$
\begin{aligned}
\Phi_{n}^{12}\left(u_{1}, u_{2}\right)= & \int_{0}^{\infty} \int_{0}^{u_{1}+c_{1} t} \Phi_{n}^{12}\left(u_{1}+c_{1} t-x_{1}, u_{2}+c_{2} t\right) f_{11}\left(x_{1}\right) \lambda_{11} e^{-\lambda_{s} t} d x_{1} d t \\
& +\int_{0}^{\infty} \int_{0}^{u_{2}+c_{2} t} \Phi_{n}^{12}\left(u_{1}+c_{1} t, u_{2}+c_{2} t-x_{2}\right) f_{22}\left(x_{2}\right) \lambda_{22} e^{-\lambda_{s} t} d x_{2} d t \\
& +\int_{0}^{\infty} \Phi_{n}^{12}\left(u_{1}+c_{1} t, u_{2}+c_{2} t\right) \lambda_{33} e^{-\lambda_{s} t} d t \\
& +\int_{0}^{\infty} \int_{0}^{\infty} \int_{0}^{u_{2}+c_{2} t} \int_{0}^{u_{1}+c_{1} t} \Phi_{n}^{12}\left(u_{1}+c_{1} t-x_{1}, u_{2}+c_{2} t-x_{2}\right) f_{c}\left(x_{1}, x_{2}, x_{3}\right) \lambda_{c} e^{-\lambda_{s} t} d x_{1} d x_{2} d x_{3} d t
\end{aligned}
$$

with starting value $\Phi_{0}^{12}\left(u_{1}, u_{2}\right)=1$. Other probabilities $\Phi_{n}^{2}\left(u_{2}\right), \Phi_{n}^{3}\left(u_{3}\right), \Phi_{n}^{13}\left(u_{1}, u_{3}\right)$ and $\Phi_{n}^{23}\left(u_{2}, u_{3}\right)$ appearing in (26) can be evaluated in the same manner.

\section{$6 \quad$ Numerical illustrations}

In this section we present numerical illustrations of the results obtained in the previous sections in the bivariate process (2) under exponential claims. The premium rates are assumed to be $c_{1}=3.2$ and $c_{2}=30$, whereas the mean claim sizes are $1 / \mu_{1}=1$ and $1 / \mu_{2}=10$. Furthermore, based on different arrival rates we consider four different bivariate risk models as follows 
- Case 1: Independent model - $\lambda_{11}=\lambda_{22}=2 ; \lambda_{12}=0$.

- Case 2: Three-component common shock model - $\lambda_{11}=\lambda_{22}=1.5 ; \lambda_{12}=0.5$.

- Case 3: Three-component common shock model - $\lambda_{11}=\lambda_{22}=0.5 ; \lambda_{12}=1.5$.

- Case 4: Common shock model - $\lambda_{11}=\lambda_{22}=0 ; \lambda_{12}=2$.

Note that under all four scenarios the total arrival rates for the companies are $\lambda_{1}=\lambda_{2}=2$, and the relative security loadings are $\theta_{1}=0.6$ and $\theta_{2}=0.5$. Under Case 1 we are dealing with two independent risk processes. The second and the third cases deal with a three-component common shock model, where in Case 2 common shocks occur less frequently than in Case 3. Finally Case 4 assumes only the existence of common shocks which generate claims in both lines of business every time. We are particularly interested to see the impact of dependence versus independence on various ruin-related quantities.

\subsection{Ruin probability and expected discounted deficit}

In this subsection we assume the two insurers possess initial surplus values $u_{1}=2$ and $u_{2}=$ 10. First, the marginal or univariate ruin probabilities can be calculated using the well-known formulas

$$
\begin{aligned}
& \Psi^{1}\left(u_{1}\right)=\frac{1}{1+\theta_{1}} e^{-\frac{\theta_{1} \mu_{1}}{1+\theta_{1}} u_{1}}=0.2952291 \quad\left(=\lim _{n \rightarrow \infty} \Psi_{n}^{1}\left(u_{1}\right)\right), \\
& \Psi^{2}\left(u_{2}\right)=\frac{1}{1+\theta_{2}} e^{-\frac{\theta_{2} \mu_{2}}{1+\theta_{2}} u_{2}}=0.4776875 \quad\left(=\lim _{n \rightarrow \infty} \Psi_{n}^{2}\left(u_{2}\right)\right) .
\end{aligned}
$$

Under the independent Case 1 , the ultimate ruin probability $\Psi^{\text {and }}\left(u_{1}, u_{2}\right)$ for $T_{\text {and }}$ is simply the product of the univariate ruin probabilities. Therefore, using similar relationship as in (24), the exact ultimate ruin probability for $T_{\text {or }}$ in Case 1 can be calculated as $\Psi\left(u_{1}, u_{2}\right)=\Psi^{1}\left(u_{1}\right)+$ $\Psi^{2}\left(u_{2}\right)-\Psi^{1}\left(u_{1}\right) \Psi^{2}\left(u_{2}\right)=0.6318894$.

\section{Insert Figure 1}

Figure 1 plots the ruin probabilities $\Psi_{n}\left(u_{1}, u_{2}\right)$ in the four cases of bivariate risk processes along with the univariate cases of $\Psi_{n}^{i}\left(u_{i}\right)$ for $i=1,2$, all against the number of claims $n$. The results are given based on 100 iterations for which the algorithms in Section 4.1 and Example 5 are used. Note that the computational times for running 100 iterations using our procedures of recursive sums are somewhere between 10 to 12 hours, which is considerably smaller than those needed for applying the recursive integrals from Example 1. From Figure 1 we can observe that as the number of iterations increases, the results converge. In the independent Case 1, after 100 iterations we obtain a ruin probability of 0.6306428 that is very close to the exact value of 0.6318894. Looking at Figure 1 we can also observe that the ultimate ruin probability of Case 1 is the highest one in all models, being followed by Cases 2-4. In this example, these numerical values suggest that if one uses the ruin probabilities associated to $T_{\text {or }}$ as a measure of riskiness, the independent Case 1 is the most risky, whereas the dangerousness decreases with the frequency 


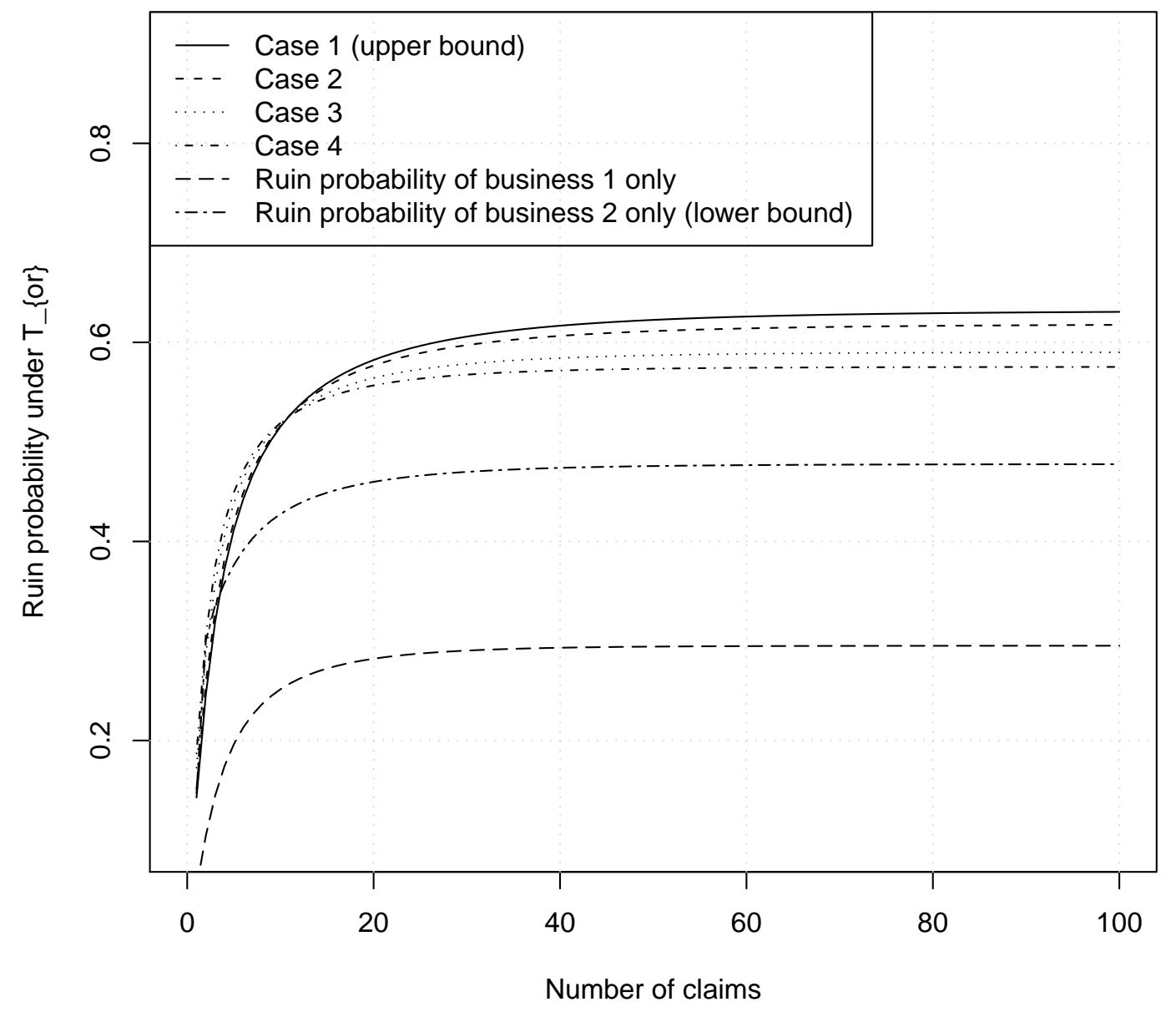

Figure 1: Ruin probabilities in $T_{\text {or }}$ for four bivariate cases and two univariate cases.

of common shocks to the processes $\left\{U_{1}(t)\right\}_{t \geq 0}$ and $\left\{U_{2}(t)\right\}_{t \geq 0}$ (keeping the total arrival rate of each company fixed).

Chan et al. (2003), Cai and Li (2005) and Yuen et al. (2006) derived bounds for the ultimate ruin probability $\Psi\left(u_{1}, u_{2}\right)$ under $T_{\text {or }}$ as

$$
\max \left\{\Psi^{1}\left(u_{1}\right), \Psi^{2}\left(u_{2}\right)\right\} \leq \Psi\left(u_{1}, u_{2}\right) \leq \Psi^{1}\left(u_{1}\right)+\Psi^{2}\left(u_{2}\right)-\Psi^{1}\left(u_{1}\right) \Psi^{2}\left(u_{2}\right),
$$

where the final expression is exactly the ruin probability in the case where $\left\{U_{1}(t)\right\}_{t \geq 0}$ and $\left\{U_{2}(t)\right\}_{t \geq 0}$ are independent. As it can be seen from Figure 1, the lower bound in (27) is not very tight compared to Cases 2, 3 and 4, whereas the upper bound given by Case 1 is relatively close to the exact ultimate ruin probabilities. One can also observe that the upper bound works better when $\left\{U_{1}(t)\right\}_{t \geq 0}$ and $\left\{U_{2}(t)\right\}_{t \geq 0}$ are less dependent. 


\section{Insert Figure 2}

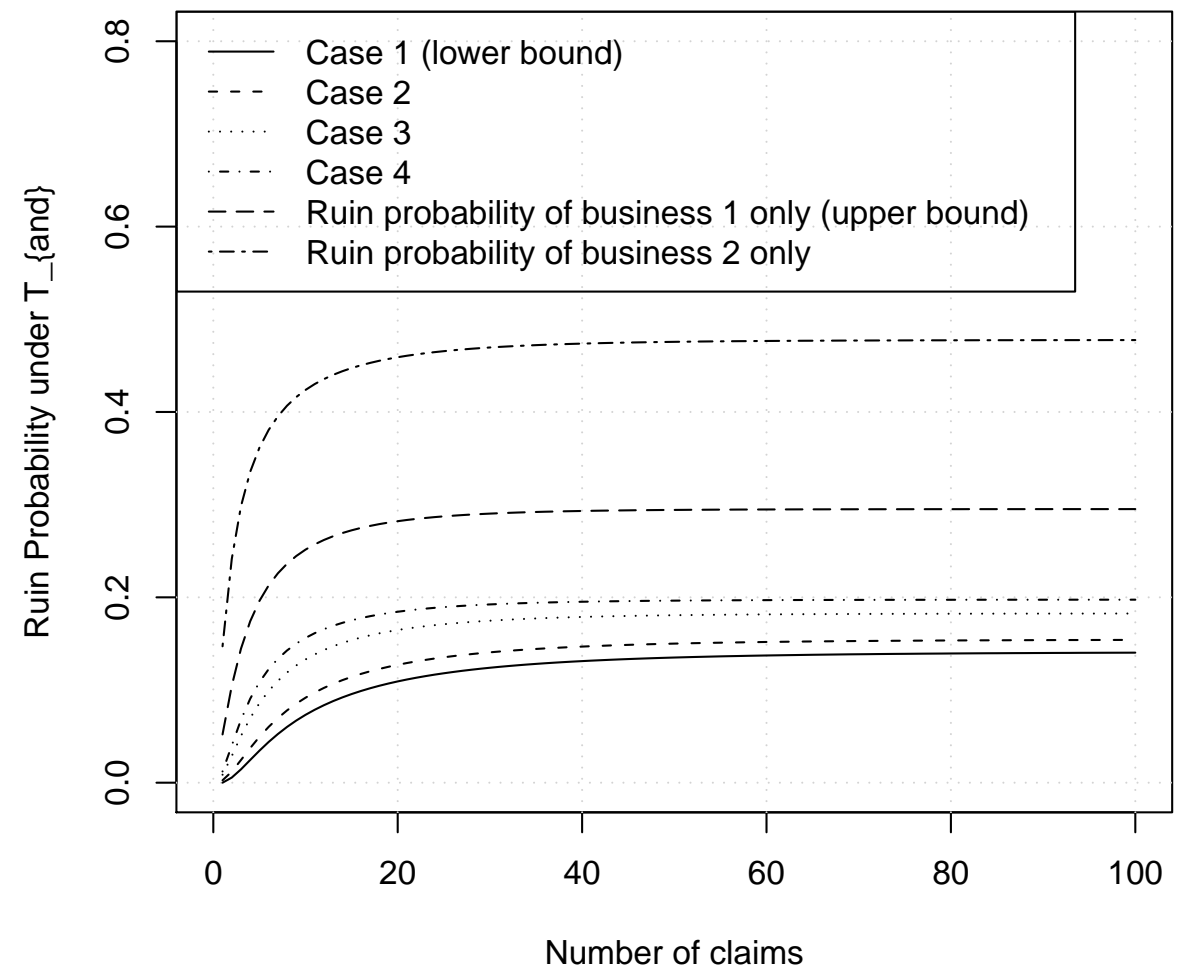

Figure 2: Ruin probabilities in $T_{\text {and }}$ for four bivariate cases and two univariate cases.

Using the results in Section 5, we further consider the ultimate ruin probability $\Psi^{\text {and }}\left(u_{1}, u_{2}\right)$ related to $T_{\text {and }}$. Cai and $\operatorname{Li}(2005,2007)$ provided simple bounds for $\Psi^{\text {and }}\left(u_{1}, u_{2}\right)$ given by

$$
\Psi^{1}\left(u_{1}\right) \Psi^{2}\left(u_{2}\right) \leq \Psi^{\text {and }}\left(u_{1}, u_{2}\right) \leq \min \left\{\Psi^{1}\left(u_{1}\right), \Psi^{2}\left(u_{2}\right)\right\} .
$$

Figure 2 displays the ruin probabilities $\Psi_{n}^{\text {and }}\left(u_{1}, u_{2}\right)$ for the four bivariate cases as well as the univariate cases. Note that the upper bound in (28) is not very tight, whereas the lower bound given by the independent Case 1 is relatively good. The lower bound works better when $\left\{U_{1}(t)\right\}_{t \geq 0}$ and $\left\{U_{2}(t)\right\}_{t \geq 0}$ are less dependent. As a general conclusion suggested by Figures 1 and 2, if one assumes independence instead of dependence via common shocks, one overestimates the ruin probability under $T_{\text {or }}$ and underestimates the ruin probability under $T_{\text {and }}$.

We also consider the expected discounted deficit at ruin as in Section 4.2 for the same cases described above. Figure 3 displays the values at a discount factor $\delta=0.05$, when ruin happens before or on the $n$-th claim, i.e. partial sums of (10) are plotted (contrary to the infinite sum in (11)) under the appropriate choice of penalty functions. The expected discounted deficit appears to converge to around 4.1 in all four cases. However, when we look closer at the actual numbers as $n$ increases (not reproduced here), they appear to converge to slightly different values. 


\section{Insert Figure 3}

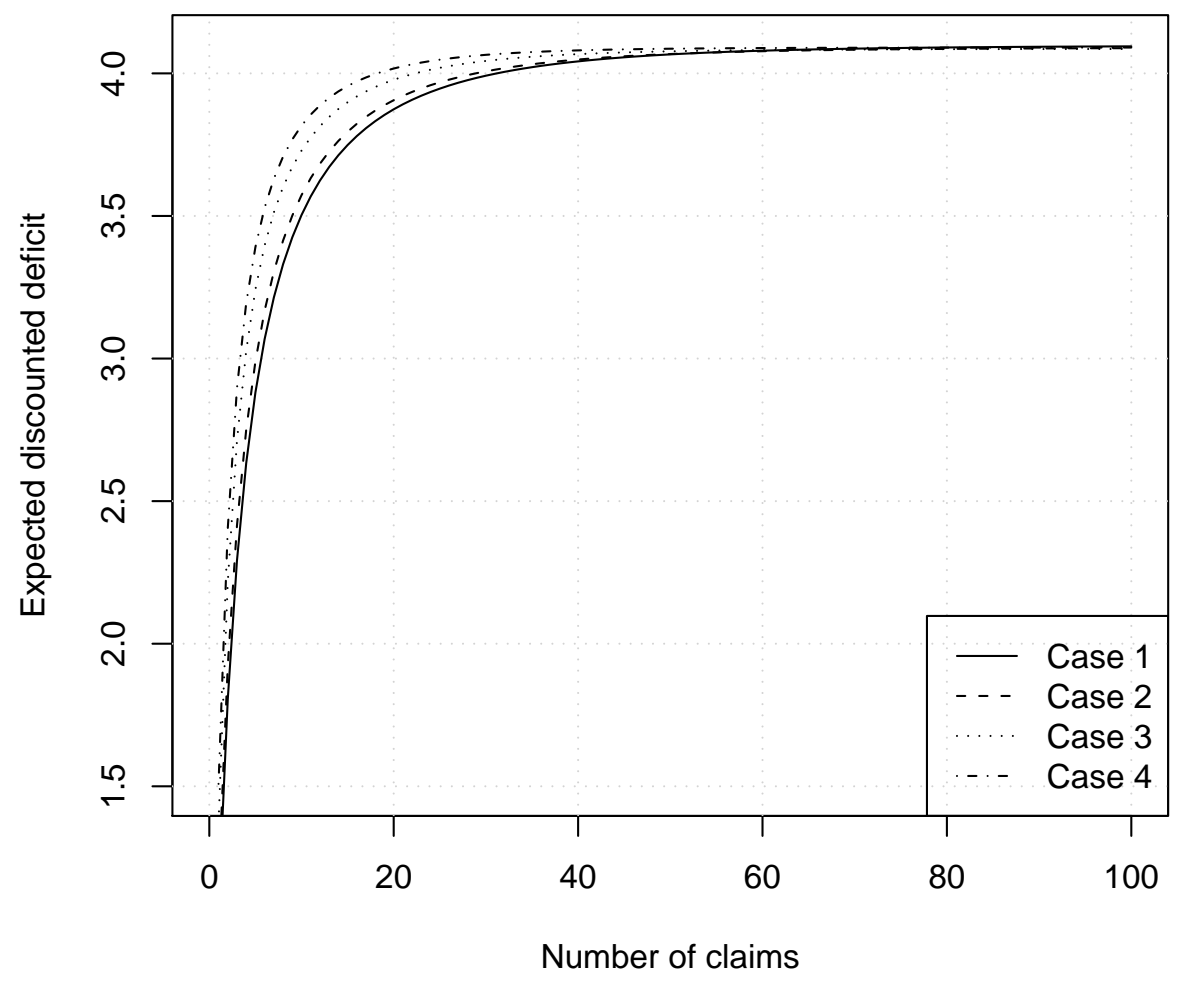

Figure 3: Expected discounted deficits up to the $n$-th claim for four different bivariate models.

\subsection{Expected discounted total surplus when only one line is ruined}

Next, we are interested in the quantity defined in (9) which represents the expected discounted total surplus of the two companies at time $T_{\text {or }}$ when business 1 is ruined, but business 2 has positive surplus. Again we assume initial surplus levels of $u_{1}=2$ and $u_{2}=10$, and a discount factor of $\delta=0.05$. Since the recursive evaluation of the coefficients in this case is very similar to those in Propositions 1 and 2, we omit the rather repetitive details here and only show the plots.

\section{Insert Figure 4}

From Figure 4, one observes that the expected discounted total surplus values are always positive under all four scenarios. The intuitive explanation is as follows. It is evident from the premium rates as well as the means of claim size in the two lines of business that the scale of line 2 is much larger than that of line 1 . Therefore, at the time when line 1 is ruined but not line 2 , there is very good chance that the positive surplus in line 2 is more than sufficient to cover 


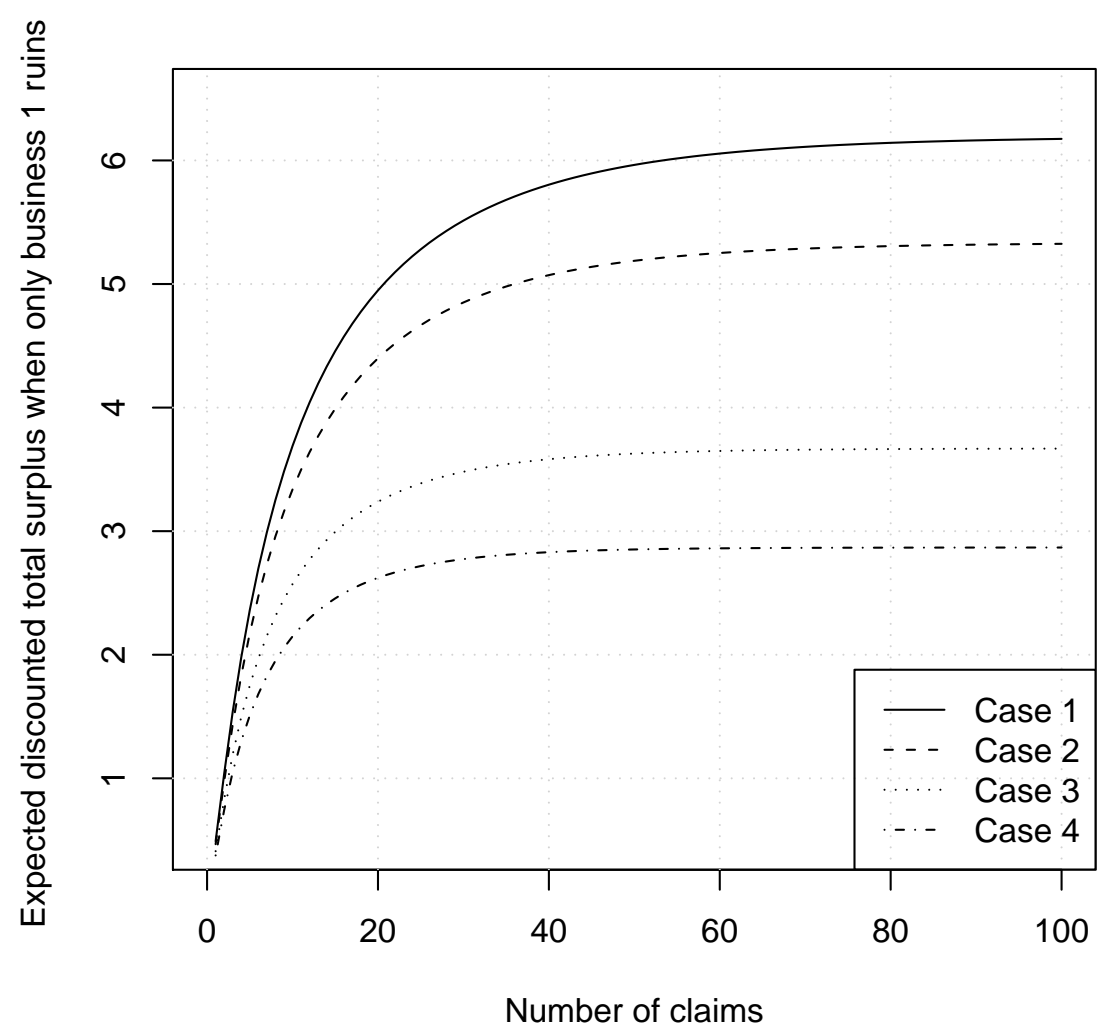

Figure 4: Expected discounted total surplus values if only business 1 is ruined up to the $n$-th claim.

the deficit in line 1, resulting in a positive total surplus. Furthermore, note that the expected discounted total surplus appears to increase as $\left\{U_{1}(t)\right\}_{t \geq 0}$ and $\left\{U_{2}(t)\right\}_{t \geq 0}$ are less dependent.

Similarly, the expected discounted total surplus of the two lines at time $T_{\text {or }}$ when line 2 is ruined but not line 1 is depicted in Figure 5. This quantity is achieved by letting $w^{\{2\}}\left(y_{1}, y_{2}\right)=$ $y_{1}+y_{2}$ and $w^{\{1\}}(\cdot, \cdot) \equiv w^{\{1,2\}}(\cdot, \cdot) \equiv 0$. This time the expected discounted total surplus values up to the $n$-th claim are always negative. Interestingly, as the number of claims increases, this quantity appears to be first decreasing sharply, then slightly increasing before finally converging. Since Figure 5 shows the partial sums of (10), the slight increase in the interim means that $\phi_{n}\left(u_{1}, u_{2}\right)$ indeed becomes slightly positive as $n$ gets large. This might be attributed to the fact that when ruin in line 2 occurs at large enough number of claims, there is good chance that line 1 has already accumulated sufficient capital or profits to cover line 2's deficit due to the long time elapsed, even its scale is smaller. Again the expected discounted total surplus appears to be larger if $\left\{U_{1}(t)\right\}_{t \geq 0}$ and $\left\{U_{2}(t)\right\}_{t \geq 0}$ are less dependent.

\section{Insert Figure 5}




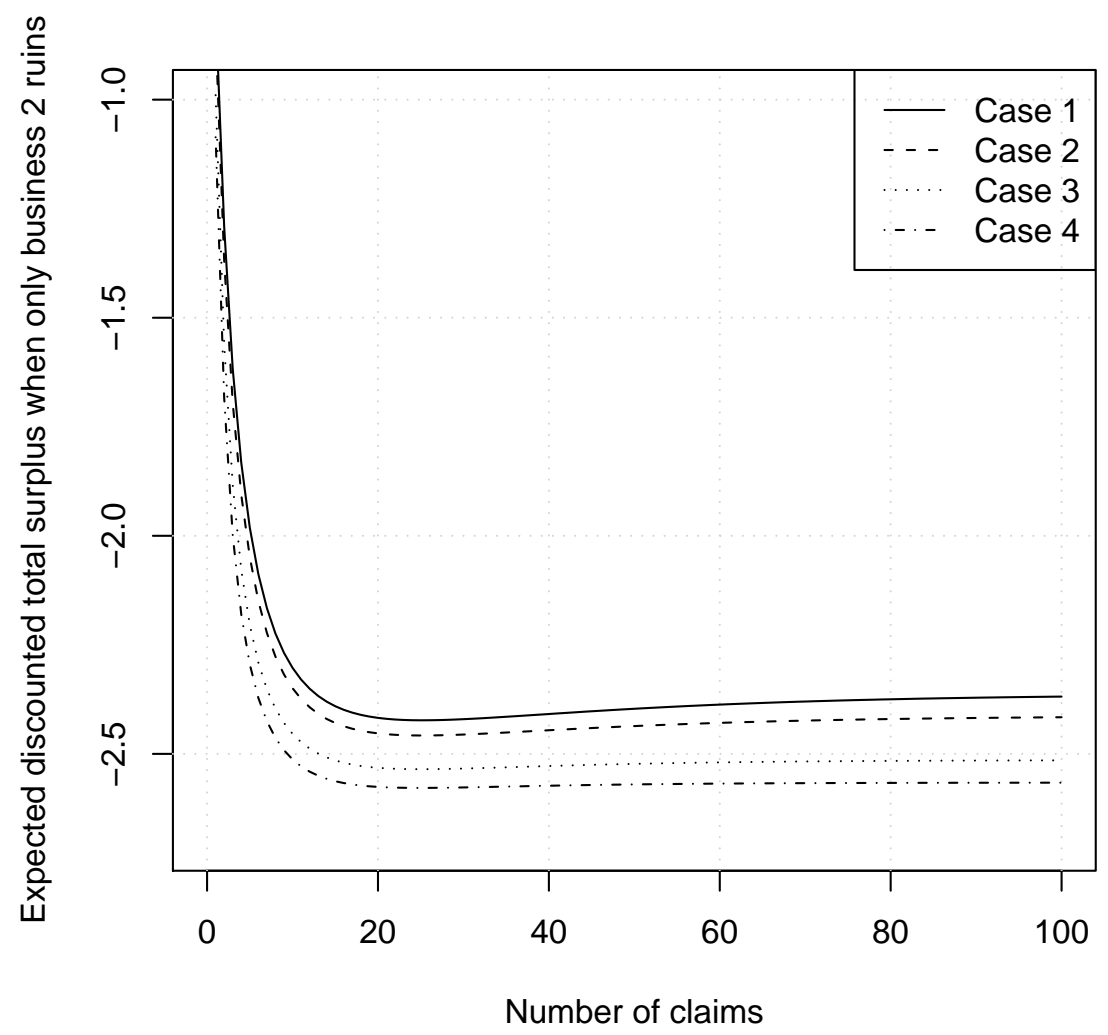

Figure 5: Expected discounted total surplus values if only business 2 is ruined up to the $n$-th claim.

\subsection{Optimal capital allocation}

In order to show the usefulness of the method of recursive sums presented in Section 4 (see Remark 2), in this subsection we study an optimal capital allocation problem formulated as follows. Suppose that a company possesses two lines of business which are to share among them a fixed amount of initial fund valued at $K$ dollars. A criterion will be needed to make a corporate decision regarding the optimal way of splitting the $K$ dollars. This can be based on risk measures derived from the bivariate process $\left\{\left(U_{1}(t), U_{2}(t)\right)\right\}_{t \geq 0}$, which include, for example, minimizing the joint ruin probability (in various definitions) or the expected discounted deficit. In this subsection, we are interested in the former problem. In the $T_{\text {or }}$ case, this means finding the optimal initial surplus values $\left(u_{1}^{*}, u_{2}^{*}\right)$ which minimize $\Psi\left(u_{1}, u_{2}\right)$ under the constraints $u_{1}+u_{2}=K$ and $u_{1}, u_{2} \geq 0$. Equivalently, we need to find the optimal value $u_{1}^{*}$ to minimize $\Psi\left(u_{1}, K-u_{1}\right)$ within the domain $0 \leq u_{1} \leq K$. The minimization of ruin probability under $T_{\text {and }}$ can be defined in the same manner.

Figures 6 and 7 plot the joint ruin probability against the initial surplus $u_{1}$ of business line 1 for the four cases of bivariate risk processes under $T_{\text {or }}$ and $T_{\text {and }}$ respectively. Here we assume that two lines of business share total initial capital of 12 (i.e. $u_{1}+u_{2}=12$ ) and other model 
parameters are not changed. The results given in this subsection are based on our algorithms of recursive sums up to the 100-th iteration, i.e. $\Psi_{100}\left(u_{1}, u_{2}\right)$ and $\Psi_{100}^{\text {and }}\left(u_{1}, u_{2}\right)$ are used instead of $\Psi\left(u_{1}, u_{2}\right)$ and $\Psi^{\text {and }}\left(u_{1}, u_{2}\right)$. As we mentioned in Section 6.1 , the results after 100 iterations are very close to the exact value. Therefore, we assume that all curves in Figures 6-8 essentially represent the exact ultimate ruin probabilities associated with $T_{\text {or }}$ or $T_{\text {and }}$.

\section{Insert Figures 6 and 7}

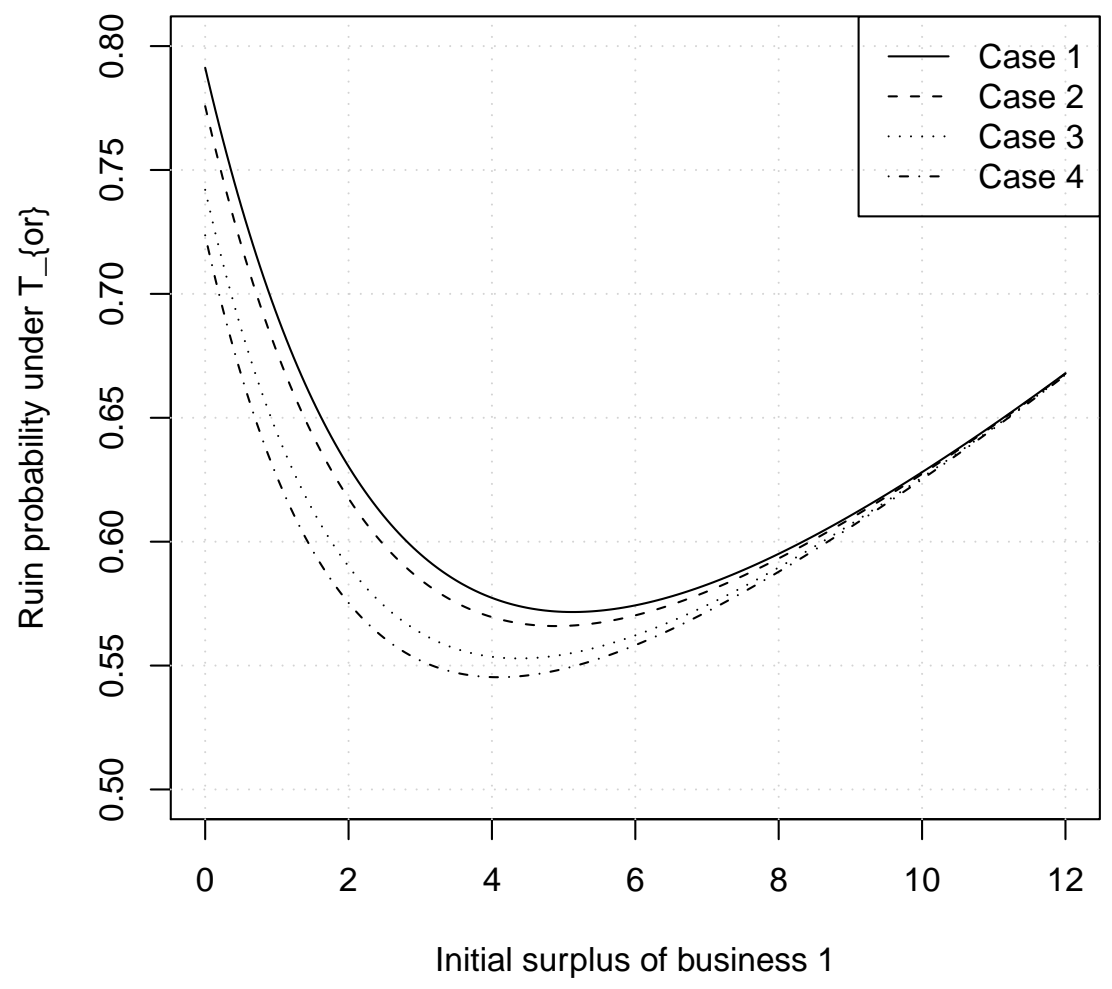

Figure 6: Optimal allocations in $T_{\text {or }}$ for four bivariate cases.

By looking at Figure 6 for $T_{\text {or }}$, we can draw the same conclusion as we did in the first numerical example in Section 6.1: the independent Case 1 is the most risky one among all models. The minimum ruin probability is 0.572 for Case 1 , followed by 0.566 for Case 2, 0.553 for Case 3 and 0.545 for Case 4 . We also observe that the insurance company needs to allocate more initial capital to line 1 in the independent case than other dependent cases in order to minimize the joint ruin probability under $T_{\text {or }}$. The weights of the total initial capital 12 assigned to line 1 are $0.4275,0.4075,0.3650$ and 0.3408 respectively for Cases $1-4$.

Compared to $T_{\text {or }}$, the results for $T_{\text {and }}$ presented in Figure 7 are quite different. The insurance company needs to put all initial capital to business line 1 to obtain the minimum ruin probability which is almost 0 in all four cases. We believe that the main reason why the optimal $u_{1}^{*}$ is always 


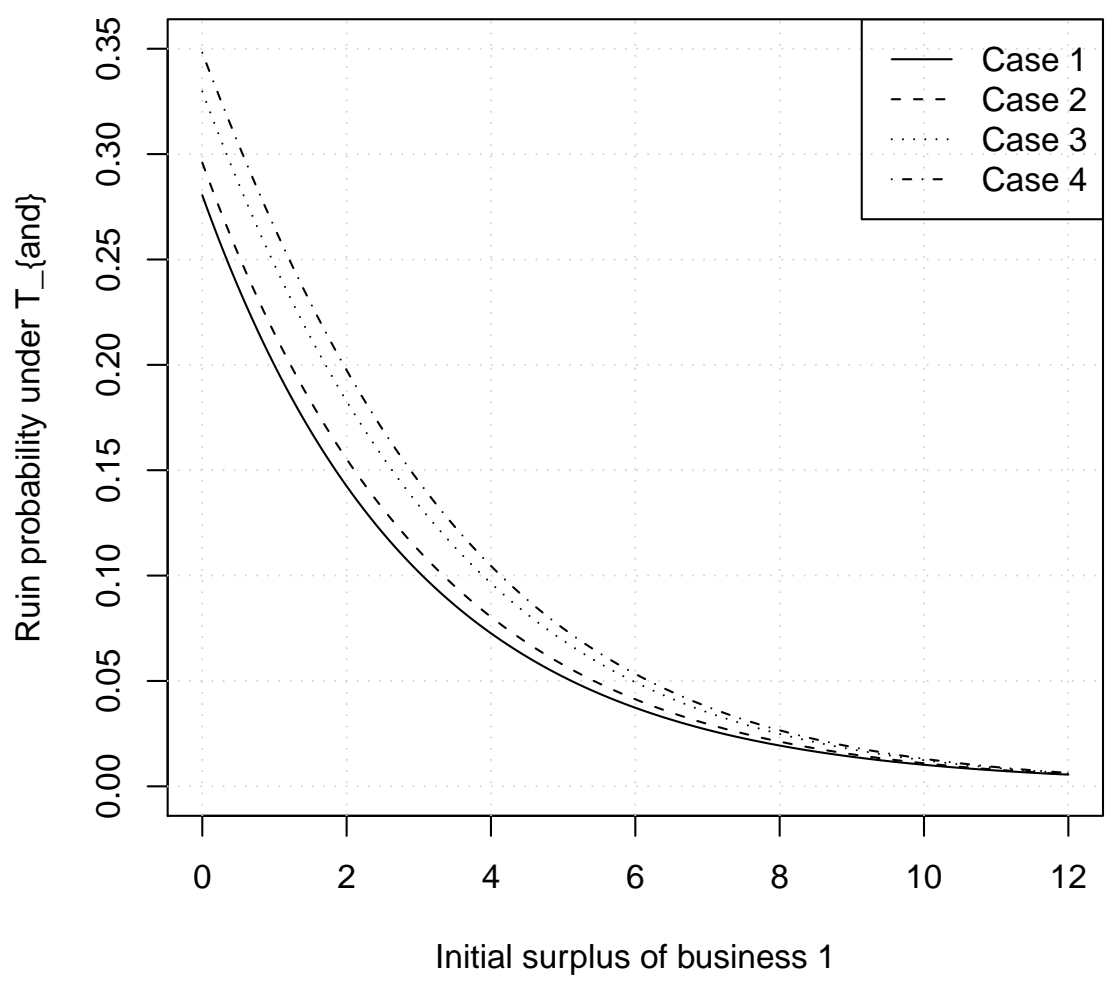

Figure 7: Optimal allocations in $T_{\text {and }}$ for four bivariate cases.

located at 12 (i.e. $100 \%$ of the total initial surplus) is that line 1 has much smaller scale than line 2. More precisely, the average claim amount for business line 1 is only $10 \%$ of that of line 2. Consequently, line 1 will survive much longer than line 2 if both lines have the same initial surplus. As we pointed out in Section $1, T_{\text {and }}$ is the later individual ruin time of the two lines, so the insurance company should invest all its limited initial capital to line 1 to minimize the joint ruin probability associated with $T_{\text {and }}$, even if this means that line 2 is likely to ruin early.

In order to show that the method of recursive sums is simple to implement in practice, we also plot the indifference curves associated to $T_{\text {or }}$ in Figure 8 for Case 2 . An indifference curve represents all the possible combinations of $u_{1}$ and $u_{2}$ which give the same ruin probability as stated on the right-hand side of the figure. If we have the budget restriction $u_{1}+u_{2}=12$, we will reach our optimal allocation result that gives $u_{1}^{*}=4.89$ and $u_{2}^{*}=7.11$.

\section{Insert Figure 8}




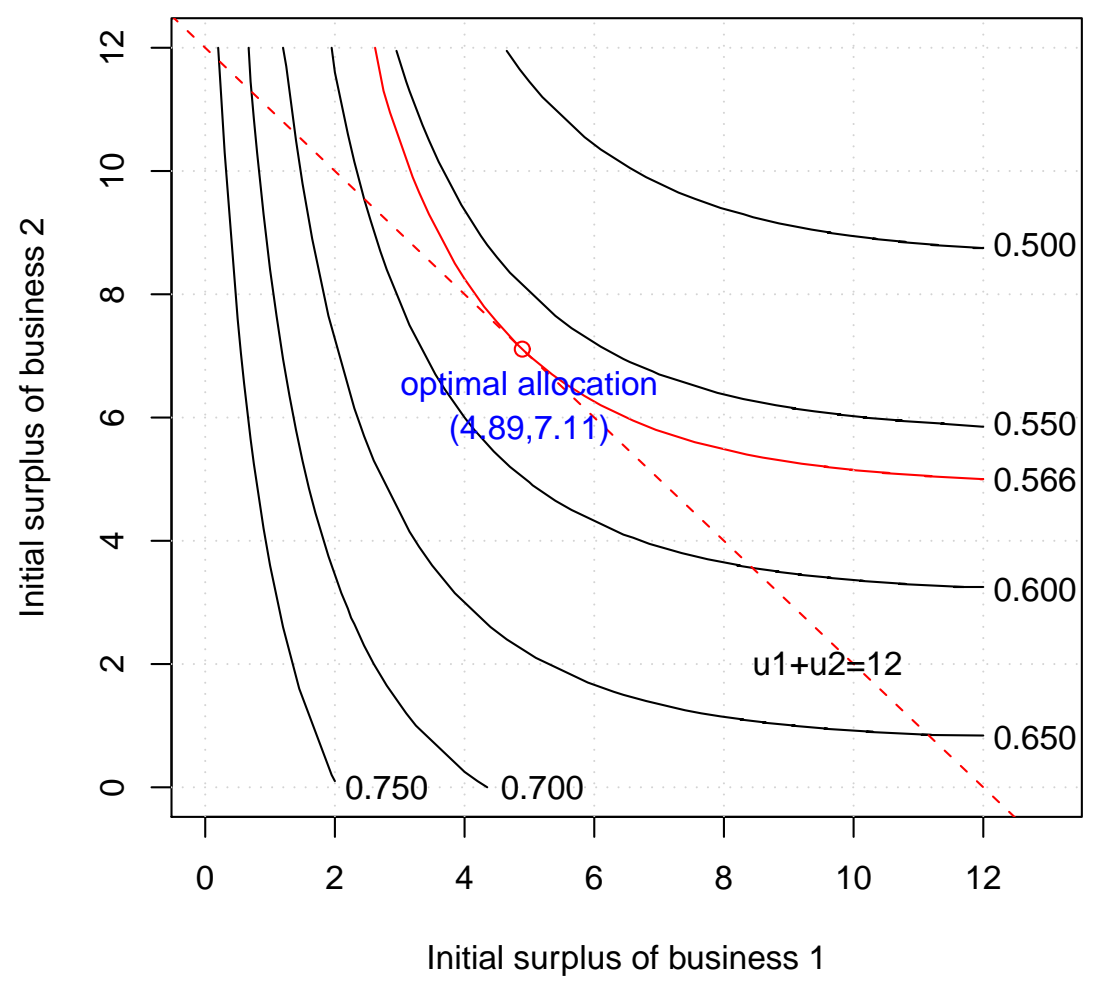

Figure 8: Indifference curves for Case 2.

\section{Conclusion}

In this paper we consider a multi-dimensional risk model with claims occurring according to Poisson processes with common shocks. Using an intuitive recursive approach that is based on observing the risk processes at claim instants, we provide recursive integrals to find the survival probabilities as well as a subclass of the Gerber-Shiu functions with a penalty that depends on the surplus levels of the all individual processes at ruin only. In Propositions 1 and 2, it is shown via examples that the multiple/recursive integrals in the general formulas can be replaced by multiple/recursive sums that are computational more tractable. As most of the papers on multidimensional risk theory provide bounds and/or asymptotic results, we believe that our result is a step forward towards solving these very difficult problems. Moreover, our results also provide a neat way to perform related study such as an optimal capital allocation problem, as illustrated in Section 6.3.

The ideas regarding the reduction of recursive integrals to recursive sums employed in Section 4 do not limit their applications to Propositions 1 and 2 about the bivariate version of model (2) in which the claims between the two lines are independent in the case of a common shock. For 
example, in the bivariate case, in order to reduce the recursive integrals to recursive sums, one may assume that the density $f_{12}(\cdot, \cdot)$ follows, for example, a bivariate exponential distribution or a Farlie-Gumbel-Morgenstern copula with Erlang marginals. In addition to relaxing assumptions on the claims arising from common shocks, the recursive techniques can also be used when one deals with renewal type risk models (as in Stanford et al. (2000)), where the inter-arrival times $\left\{V_{k}\right\}_{k=1}^{\infty}$ between claim events may follow, for example, Erlang or Coxian distribution (in contrast to exponential random variables with mean $\left.1 / \lambda_{s}\right)$. However, in such case we require the definition of the probabilities $\left\{\pi_{11}, \pi_{22}, \ldots, \pi_{M M}, \pi_{c}\right\}$ such that, given that a claim event occurs, with probability $\pi_{i i}(i=1,2, \ldots, M)$ there is a claim in line $i$ but not in any other lines, and with probability $\pi_{c}$ it is a common shock causing claims in all lines at the same time. Moreover, one may also allow the inter-arrival times $\left\{V_{k}\right\}_{k=1}^{\infty}$ and the resulting claims to be dependent, with dependency structure resembling the ones studied by Boudreault et al. (2006) or Cossette et al. (2008). As the ideas and techniques involved in the derivations of the afore-mentioned generalizations are essentially the same as those in the simpler examples presented in this paper, we restrict ourselves solely to mentioning these cases, leaving them as rather tedious algebraic exercises.

Note that in the present model (1), it is assumed that the common shock affects all lines simultaneously. We remark that the model can be further extended to allow for multiple common shock components such that each component induces claims in a different subset of the $M$ lines. Again, such presentation will be notationally involved without much gain of additional insights, so we have opted to study (1) for ease of presentation.

As mentioned by most authors, ruin theory in the multi-dimensional case is very complex. Our method is rather tedious, but we use only elementary algebra to provide a way of calculating various ruin-related quantities under a multi-dimensional risk model. We hope that this paper will stimulate more research on these complicated risk models that seem to become more popular in the literature.

Acknowledgement: The authors would like to thank the anonymous referee for his/her helpful comments and suggestions which greatly improved an earlier version of the paper. Andrei Badescu gratefully acknowledges financial support received from the Natural Sciences and Engineering Research Council of Canada (NSERC). Support for Eric C.K. Cheung from a start-up fund provided by the Faculty of Science and the Department of Statistics and Actuarial Science as well as the Seed Funding for Basic Research (Project number: 201103159001) provided by the University Research Committee at the University of Hong Kong is also gratefully acknowledged.

\section{References}

[1] Asmussen, S. and Albrecher, H. 2010. Ruin Probabilities. Second Edition. New Jersey: World Scientific.

[2] Avram, F., Palmowski, Z. and Pistoris, M. 2008a. A two-dimensional ruin problem on the positive quadrant. Insurance: Mathematics and Economics 42(1): 227-234. 
[3] Avram, F., Palmowski, Z. and Pistoris, M. 2008b. Exit problem of a two-dimensional risk process from the quadrant: Exact and asymptotic results. Annals of Applied Probability 18(6): 2421-2449.

[4] Badescu, A.L., Cheung, E.C.K. and Rabehasaina, L. 2011. A two-dimensional risk model with proportional reinsurance. Journal of Applied Probability 48(3). To appear.

[5] Boudreault, M., Cossette, H., Landriault, D. and Marceau, E. 2006. On a risk model with dependence between interclaim arrivals and claim sizes. Scandinavian Actuarial Journal (5): $265-285$.

[6] Cai, J. and Li, H. 2005. Multivariate risk model of phase type. Insurance: Mathematics and Economics 36(2): 137-152.

[7] Cai, J. and Li, H. 2007. Dependence properties and bounds for ruin probabilities in multivariate compound risk models. Journal of Multivariate Analysis 98(4): 757-773.

[8] Chan, W.-S., Yang, H. and Zhang, L. 2003. Some results on the ruin probability in a twodimensional risk model. Insurance: Mathematics and Economics 32(3): 345-358.

[9] Collamore, J.F. 1996. Hitting probabilities and large deviations. The Annals of Probability 24(4): 2065-2078.

[10] Collamore, J.F. 1998. First passage times of general sequences of random vectors: A large deviations approach. Stochastic Processes and their Applications 78(1): 97-130.

[11] Collamore, J.F. 2002. Importance sampling techniques for the multidimensional ruin problem for general Markov additive sequences of random vectors. The Annals of Applied Probability 12(1): $382-421$.

[12] Cossette, H., Marceau, E. and Marri, F. 2008. On the compound Poisson risk model with dependence based on a generalized Farlie-Gumbel-Morgenstern copula. Insurance: Mathematics and Economics 43(3): 444-455.

[13] Czarna, I., Palmowski, Z. 2011. De Finetti's dividend problem and impulse control for a two-dimensional insurance risk process. Stochastic Models 27(2): 220-250.

[14] Dang, L., Zhu, N. and Zhang, H. 2009. Survival probability for a two-dimensional risk model. Insurance: Mathematics and Economics 44(3): 491-496.

[15] Gerber, H.U. and Shiu, E.S.W. 1998. On the time value of ruin. North American Actuarial Journal 2(1): 48-72.

[16] Lee, S.C.K. and Lin, X.S. 2010. Modeling and evaluating insurance losses via mixtures of Erlang distributions. North American Actuarial Journal 14(1): 107-130.

[17] Li, J., Liu, Z. and Tang, Q. 2007. On the ruin probabilities of a bidimensional perturbed risk model. Insurance: Mathematics and Economics 41(1): 185-195.

[18] Rabehasaina, L. 2009. Risk processes with interest force in Markovian environment. Stochastic Models 25(4): 580-613. 
[19] Stanford, D.A. and Stroiński, K.J. 1994. Recursive methods for computing finite-time ruin probabilities for phase-type distributed claim sizes. ASTIN Bulletin 24(2): 235-254.

[20] Stanford, D.A., Stroiński, K.J. and Lee, K. 2000. Ruin probabilities based at claim instants for some non-Poisson claim processes. Insurance: Mathematics and Economics 26(2-3): 251267.

[21] Willmot, G.E. and Woo, J.-K. 2007. On the class of Erlang mixtures with theoretic applications. North American Actuarial Journal 11(2): 99-115.

[22] Yuen, K.C., Guo, J. and Wu, X. 2006. On the first time of ruin in the bivariate compund Poisson model. Insurance: Mathematics and Economics 38(2): 298-308. 